\title{
Patterning the embryonic pulmonary mesenchyme
}

Katharine Goodwin ${ }^{1}$, Jacob M. Jaslove ${ }^{2,3}$, Hirotaka Tao ${ }^{4}$, Min Zhu $^{4,5}$, Sevan Hopyan ${ }^{4,6,7}$, and Celeste M. Nelson ${ }^{2,8^{*}}$

1. Lewis-Sigler Institute for Integrative Genomics, Princeton University, Princeton, NJ 08544, USA

2. Department of Molecular Biology, Princeton University, Princeton, NJ 08544, USA

3. Graduate School of Biomedical Sciences, Rutgers Robert Wood Johnson Medical School, Piscataway, NJ 08854, USA

4. Program in Developmental and Stem Cell Biology, Research Institute, The Hospital for Sick Children, Toronto, ON, Canada M5G 0A4

5. Department of Mechanical and Industrial Engineering, University of Toronto, M5S 3G8

6. Department of Molecular Genetics, University of Toronto, M5S 1A8

7. Division of Orthopaedic Surgery, Hospital for Sick Children and University of Toronto, M5G 1X8

8. Department of Chemical and Biological Engineering, Princeton University, Princeton, NJ 08544, USA

(*) Address correspondence to C.M.N.

303 Hoyt Laboratory, William Street

Princeton University

Princeton, NJ 08544

Tel: 609-258-8851

Fax: 609-258-1247

E-mail: celesten@princeton.edu

Abbreviations: DC, diffusion component; Fgf, fibroblast growth factor; Foxp1, Forkhead Box P1; Gli1, glioma-associated oncogene 1; Hoxb6, homeobox B6; Lef1, Lymphoid Enhancer Binding Factor 1; MGI, Mouse Genome Informatics; Myocd, myocardin; Shh, sonic hedgehog, SMA, smooth muscle actin; SRF, serum response factor; TGF, transforming growth factor; TTF1, thyroid transcription factor 1; UMAP, uniform manifold approximation and projection; Wnt, wingless-Type MMTV Integration Site Family; WT1, Wilms tumor 1 


\begin{abstract}
Smooth muscle guides morphogenesis of epithelia during development of several organs, including the mammalian lung. However, it remains unclear how airway smooth-muscle differentiation is spatiotemporally patterned and whether it originates from distinct mesenchymal progenitors. Using single-cell RNA-sequencing of embryonic mouse lungs, we show that the pulmonary mesenchyme contains a continuum of cell identities, but no distinct progenitors. Transcriptional variability correlates with sub-epithelial and sub-mesothelial mesenchymal compartments that are regulated by Wnt signaling. Live-imaging and tension sensors reveal patterned migratory behaviors and cortical forces in each compartment, and show that subepithelial mesenchyme gives rise to airway smooth muscle. Differentiation trajectory reconstruction reveals that cytoskeleton, adhesion, and Wnt signaling pathways are activated early in differentiation. Finally, we show that Wnt activation stimulates the earliest stages of differentiation and induces local accumulation of mesenchymal F-actin, which influences epithelial morphology. Our work provides the first single-cell view of pulmonary mesenchymal patterning during branching morphogenesis.
\end{abstract}




\section{Introduction}

Smooth muscle and similar contractile cell types influence the morphogenesis of epithelial tissues $^{1-3}$. Branching of the mammalian airway epithelium has long been thought to involve smooth-muscle differentiation from the surrounding pulmonary mesenchyme ${ }^{4-8}$. Accordingly, ex vivo culture revealed that patterned airway smooth-muscle differentiation specifies cleft sites to promote epithelial bifurcation and shapes the domain branches that establish the underlying architecture of the murine lung ${ }^{9,10}$. It is therefore surprising that the smooth-muscle transcription factor myocardin $(M y o c d)$ is dispensable for lung branching morphogenesis ${ }^{11}$ and that mice lacking $\alpha$-smooth muscle actin ( $\alpha$ SMA or Acta 2$)$ are viable and only develop deleterious phenotypes in smooth muscle and similar tissues under conditions of high mechanical stress ${ }^{12,13}$. These studies reveal the complexity (and robustness) of smooth-muscle differentiation and transcriptional identity during development and highlight the importance of uncovering the factors that define a smooth-muscle cell and control its differentiation.

Lung development is intricately regulated by signaling between the epithelium, mesothelium, and mesenchyme. Shh signaling from the epithelium is required for airway smooth-muscle differentiation $^{14}$, while Fgf9 signaling from the mesothelium prevents differentiation ${ }^{15}$. The physical layout of the tissue and the differing diffusible signals from the epithelium and mesothelium could potentially establish separate mesenchymal compartments. In particular, it appears that epithelial and mesothelial signals directly influence the behavior of the subepithelial and sub-mesothelial mesenchyme, respectively ${ }^{16,17}$. 
Cells from different locations within the pulmonary mesenchyme possess different capacities for differentiation: mesenchyme that is transplanted from stalk to tip regions fails to migrate towards the epithelium or differentiate into smooth muscle unless it is exposed to Wnt $1^{18}$. Wnt signaling may therefore be involved in a compartment-specific manner in specifying smoothmuscle progenitor identity and behavior. Consistently, Wnt $2 a$ is expressed in the distal mesenchyme ${ }^{19}$ and the expression of the Axin2 is strongest in the mesenchyme around branch $\operatorname{tips}^{20}$. Deleting Ctnnbl ( $\beta$-catenin) from the mesenchyme delays branching morphogenesis and inhibits mesenchymal growth and survival ${ }^{17,21,22}$. Expressing stabilized $\beta$-catenin leads to disorganized smooth-muscle wrapping ${ }^{18}$, and Wnt 2 promotes the expression of myogenic transcription factors to support robust smooth muscle differentiation ${ }^{23}$.

Lineage-tracing studies based on single marker genes have not identified a distinct airway smooth-muscle progenitor ${ }^{24,25}$. Additionally, the signals required for patterned smooth-muscle differentiation remain unclear. Multiple pathways have been implicated but studying each one individually will not reveal how they interact to achieve spatiotemporal control. To circumvent these problems, we used single-cell RNA-sequencing (scRNA-seq) analysis. Since smooth muscle differentiates continuously during lung branching morphogenesis ${ }^{3}$, scRNA-seq analysis at a single snapshot in time can be used to interrogate cell identities ranging from undifferentiated progenitor to mature smooth muscle ${ }^{26,27}$.

ScRNA-seq analysis of E11.5 mouse lungs revealed a continuum of mesenchymal and smooth-muscle cell identities, but no distinct smooth-muscle progenitor population. Instead, 
mesenchymal cell heterogeneity reflects spatially distinct, Wnt-dependent sub-epithelial and submesothelial compartments. Live-imaging and tension-sensor experiments revealed differences in motility and cortical tension between compartments, and showed that airway smooth-muscle cells arise from the sub-epithelial mesenchyme. Computationally reconstructing this differentiation trajectory showed that cytoskeletal, adhesion, and Wnt signaling genes are upregulated early during differentiation, and that the airway smooth-muscle gene-expression program is largely Myocd-independent. Our computational analyses uncovered a role for Yap1 in mesenchymal patterning and showed that differentiating cells systematically downregulate genes involved in proliferative metabolism. Finally, we found that activated Wnt signaling causes an enrichment of nascent smooth-muscle cells and F-actin in the mesenchyme and prevents epithelial branching. Overall, we provide the first single-cell view of airway smooth-muscle differentiation and demonstrate that the earliest steps of differentiation involve Wnt-dependent mesenchymal stiffening that shapes epithelial branches.

\section{Results}

\section{scRNA-seq analysis reveals expected populations of cells in the embryonic murine lung}

We carried out scRNA-seq using cells isolated from the left lobes of lungs of CD1 mouse embryos harvested at E11.5 and analysed the data with Seurat ${ }^{28}$ (Fig. 1a, Supplementary Fig. 1a-g). After removing contaminating cells and correcting for cell-cycle stage (Supplementary Fig. 1h-k), clustering identified 6 populations of cells, each containing cells from both replicates

(Fig. 1b-c).We identified mesenchymal clusters expressing homeobox B6 (Hoxb6) and pleiotrophin (Ptn), a smooth-muscle cluster expressing Acta2, an epithelial cluster expressing 
thyroid transcription factor 1 (TTF-1 or $N k x 2.1)$, a vascular-endothelial cluster expressing VEcadherin (Cdh5), and a mesothelial cluster expressing Wt1 (Fig. 1b-e, Supplementary Table 1).

We then isolated and reclustered the closely-related mesenchymal and smooth-muscle populations, and found that the Hoxb6-, Ptn-, and Acta2-expressing cells still clustered together in a similar pattern (Supplementary Fig. 2a-b). Previous studies used lineage-tracing of Axin2-, Fgf10-, Gli1-, and Wt1-expressing cells in an attempt to identify smooth-muscle progenitors ${ }^{24,25}$. However, none of these markers labeled a distinct population in our dataset (Supplementary Fig. 2d-g). Further analysis of reclustered mesenchymal cells provided no additional evidence for a distinct smooth-muscle progenitor (Supplementary Fig. 2h-q). Similar to other developing tissues $^{29,30}$, the embryonic pulmonary mesenchyme does not harbor a distinct progenitor population, but contains of a continuum of cell states.

\section{Undifferentiated mesenchymal-cell clusters represent spatially distinct populations}

To gain insight into mesenchymal patterning, we examined the expression of markers that distinguished clusters 0 and 1 (Supplementary Table 2). Immunofluorescence analysis of $E 11.5$ lungs showed that Lef $1^{+}$cells are present adjacent to the epithelium, suggesting that these subepithelial cells may represent cluster 0 (Fig. 2a). Conversely, Foxp $1^{+}$cells are mostly in submesothelial regions and in the medial mesenchyme, suggesting that these cells comprise cluster 1 (Fig. 2b). Apart from their differential expression patterns in the mesenchyme, Lef1 and Foxp1 are detected in airway smooth muscle (Fig 2c, Supplementary Fig. 3a-b), and Foxp1 is detected in the epithelium and mesothelium (Fig 2b-c). These data suggest that the computationally 
identified mesenchymal clusters represent spatially distinct populations within the embryonic pulmonary mesenchyme. Therefore, we delineated two mesenchymal compartments, hereafter referred to as sub-epithelial (cluster 0) and sub-mesothelial (cluster 1), following previously used nomenclature ${ }^{16,17}$ (Fig. 2d).

To further validate our findings, we took advantage of published in situ hybridization results available via the Mouse Genome Informatics (MGI) database. We downloaded a list of genes that are detected in the pulmonary mesenchyme from $E 11.5$ to $E 12.5$ and classified them by expression pattern (Supplementary Table 3). We then focused on those that were clearly subepithelial or sub-mesothelial and that were detected at sufficient levels in our scRNA-seq dataset. Unsupervised clustering of mesenchymal cells based on their expression of region-specific genes showed that sub-epithelial cells group together and have similar expression of sub-epithelial markers and, likewise, that sub-mesothelial cells group together and have similar expression of their corresponding markers (Fig. 2e). UMAPs color-coded according to the summed expression of either sub-epithelial or sub-mesothelial genes show that the two sets of markers are expressed by cells in complementary regions of the UMAP (Fig. 2f). We conclude that our computationally identified clusters represent spatially distinct compartments.

We next asked whether these mesenchymal compartments persist over the pseudoglandular stage of lung development. Lef $1^{+}$cells are detected in the sub-epithelial mesenchyme around branch tips and Foxp $1^{+}$cells are detected in the sub-mesothelial mesenchyme at $E 12.5$ and $E 14.5$ (Supplementary Fig. 3c-e). Individual Foxp1 ${ }^{+}$cells are also visible in the mesenchyme in 
between branches, where they likely represent vascular endothelial cells (Fig. 2c,

Supplementary Fig. 3d, f). Therefore, the lung maintains a sub-epithelial, Lef1 ${ }^{+}$mesenchymal population and a sub-mesothelial, Foxp1-mesenchymal population over the course of branching morphogenesis. The mesenchyme in between branches (with low Lef1 and Foxp1 immunostaining) may take on new characteristics, perhaps to support formation of vasculature.

\section{Wnt signaling regulates cell identity in the embryonic pulmonary mesenchyme}

We hypothesized that the mesenchymal populations identified in our scRNA-seq dataset represent cell types with important functions in branching morphogenesis. Indeed, GO enrichment analysis of genes upregulated in each mesenchymal cluster revealed that both clusters are enriched for genes involved in "morphogenesis of a branching epithelium" (Fig. 3a, Supplementary Tables 2, 4). Additionally, our analysis revealed a significant enrichment of genes with GO terms related to Wnt signaling. We therefore examined differentially-expressed Wnt signaling-related genes (Fig. 3b). Among the genes upregulated in the sub-epithelial cluster were Lef1, a Wnt signaling effector ${ }^{31}$, and Wnt2, a Wnt ligand implicated in airway smoothmuscle differentiation ${ }^{23}$. Overall, the sub-epithelial cluster expresses more activators and targets of Wnt signaling, while the sub-mesothelial cluster expresses more inhibitors. Each mesenchymal cluster is therefore characterized by a distinct Wnt-signaling signature and, consistent with previous studies ${ }^{19,20,32}$, the sub-epithelial cluster may be more Wnt-active.

Signals from the epithelium and mesothelium have previously been shown to regulate behaviors of the sub-epithelial and sub-mesothelial mesenchyme, respectively ${ }^{16,17}$. We therefore 
hypothesized that Wnt signals from these tissues could establish compartments of differential Wnt signaling across the epithelium-to-mesothelium axis. Tissue-specific analysis using our scRNA-seq dataset confirmed that the epithelium expresses $W n t 7 b$ and the sub-epithelial mesenchyme expresses $W n t 2^{19}$ (Fig. 3c). The sub-mesothelial mesenchyme expresses Wnt11, the mesothelium expresses Wnt9a, and both tissues express the inhibitors Sfrpl and Sfrp2, suggesting that Wnt signaling may be inhibited at the mesothelial end of the axis (Fig. 3c). Vascular-endothelial and smooth-muscle cells do not show any striking expression patterns of Wnt ligands or secreted inhibitors. $F z d 1$ and $F z d 2$ are the only Wnt receptors detected in the mesenchyme, but they are not preferentially enriched in either cluster (Fig. 3c), suggesting that differential Wnt signaling across the mesenchyme arises from patterned expression of ligands and inhibitors.

To determine whether Wnt signaling can regulate the compartments of the pulmonary mesenchyme, we manipulated this pathway in lungs explanted from E11.5 CD1 mouse embryos. To activate Wnt signaling, we inhibited GSK3 by treating lung explants with $\mathrm{LiCl}^{20}$, which maintains nuclear $\beta$-catenin levels and increases cytoplasmic Axin2 intensity in the mesenchyme (Supplementary Fig. 4). Conversely, to downregulate Wnt signaling, we treated explants with IWR1, which stabilizes Axin2 leading to enhanced activity of the $\beta$-catenin destruction $\operatorname{complex}^{20}$. As expected given its mechanism of action, IWR1 treatment does not affect Axin2 intensity, but reduces nuclear $\beta$-catenin intensity in the mesenchyme (Supplementary Fig. 4). 
We then quantified the domains of Lef1 ${ }^{+}$sub-epithelial cells and Foxp $1^{+}$sub-mesothelial cells. We plotted Lef1-intensity profiles as a function of distance from the tip of the epithelial branch L.L2 (the second domain branch of the left lobe, which initiates and extends during the culture period $^{9,33}$ ) and Foxp1-intensity profiles as a function of distance from the mesothelium. LiCl-treated explants show an expanded domain of Lef $1^{+}$cells around epithelial branch tips and fewer Foxp $1^{+}$cells than DMSO-treated controls (Fig. 3d-g). Conversely, IWR1-treated explants exhibited a signficant reduction in Lef1 intensity and a random distribution of Foxp $1^{+}$cells in the mesenchyme adjacent to the tip of branch L.L2 (Fig. 3d-g). Overall, activating Wnt signaling expands the sub-epithelial mesenchyme at the expense of the sub-mesothelial mesenchyme, and inhibiting Wnt shrinks the sub-epithelial compartment. Consistently, enhancing Wnt signaling via mesenchymal deletion of the Wnt-inhibitor Adenomatous polyposis coli $(A p c)$ results in increased expression of the sub-epithelial mesenchymal marker versican $(V c a n)^{22}$ and inhibiting Wnt signaling via mesenchymal deletion of Ctnnbl results in decreased expression of the subepithelial mesenchymal markers Lef1 and cyclin D1 ${ }^{21}$ (Ccnd1; Supplementary Table 2).

To determine whether mesenchymal patterning also depends on Shh, we treated explants with the Shh antagonist cyclopamine, which prevents smooth-muscle differentiation and decreases sub-epithelial mesenchymal cell proliferation ${ }^{9,16}$. In the presence of cyclopamine, the Lef $1^{+}$, sub-epithelial domain is reduced around branch tips, but the Foxp $1^{+}$sub-mesothelial domain is slightly expanded (Fig 3h-k). We then asked whether Wnt or Shh could override each other's compartment-specific effects. We combined cyclopamine and $\mathrm{LiCl}$ treatments and found that activating Wnt signaling rescues the loss of Lef $1^{+}$cells caused by Shh inhibition (Fig. 3l, n). However, Foxp1 intensity is much lower than controls and similar to $\mathrm{LiCl}$ treatment alone (Fig. 
3m, o), suggesting that the positive effects of Shh inhibition on this compartment are indirect and cannot overcome the effects of activated Wnt signaling.

\section{Mesenchymal cell motility and cortical forces are spatially patterned during airway branching morphogenesis}

Our observations thus far suggest that the two mesenchymal clusters represent a continuum of cell states that can be shifted by Wnt signaling instead of two distinct tissues. We therefore investigated whether mesenchymal cells transition between compartments and/or into the smooth-muscle lineage. We used Dermo1-Cre driven expression of R26R-Confetti to sparsely label mesenchymal cells and performed time-lapse imaging analysis of lung explants isolated at E11.5. Dermo1-Cre is active throughout the pulmonary mesenchyme ${ }^{16}$ (Supplementary Fig. 5a), including in Lef $1^{+}$sub-epithelial and Foxp $1^{+}$sub-mesothelial mesenchymal cells (Fig. 4ab). Confetti-labelled cells were observed to elongate around epithelial buds (Supplementary

Fig. 5b) and could contribute to the smooth-muscle layer (Fig. 4c). Strikingly, Confetti-labelled cells are not clustered within groups of clones, suggesting that mesenchymal cells rearrange extensively during branching morphogenesis (Supplementary Fig. 5b). Indeed, mesenchymal cells are suprisingly motile (Supplementary Video 1), and cell tracking and mean squared displacement (MSD) analyses showed that mesenchymal cells exhibit a range of migratory behaviors, from sub-diffusive to directed (Supplementary Fig. 5c-h). Migration speeds are highly variable and are greatest among sub-epithelial mesenchymal cells (Fig. 4d), demonstrating that cell motility differs between mesenchymal compartments. 
We observed that mesenchymal cells can move between compartments over short timescales ( $\sim 5$ hours) relative to the timescales of branching in ex vivo culture ( $\sim 20$ hours). The majority stay within their compartments over the timescales analyzed, but a fraction of cells cross over into the opposite compartment (Fig. 4e-h), possibly contributing to the continuum of mesenchymal cell transcriptional states. Most tracked smooth-muscle cells were already elongated around the epithelium, but some originated from rounder, sub-epithelial cells at branch tips (Fig. 4h-j). Consistent with previous work in fixed tissues ${ }^{18}$, our time-lapse data show that smooth-muscle cells arise from the sub-epithelial mesenchyme.

Cell migration and rearrangements are regulated by cortical tension, which can be estimated using the FRET-based vinculin tension sensor (VinTS) ${ }^{34}$ (Fig. 4k). A VinTS knock-in mouse was recently generated and validated to infer cortical forces in mandibular arch morphogenesis ${ }^{35}$. Live-imaging and fluorescence lifetime imaging microscopy (FLIM) of E12.5 VinTS lungs revealed that the epithelium and sub-epithelial mesenchyme had the longest average fluorescence lifetimes (highest tension), while the vasculature had the lowest (Supplementary Fig. 5i-j). Consistent with the observed differences in migratory behavior ${ }^{35}$, we found that sub-epithelial mesenchymal cells had higher and more variable fluorescence lifetimes than sub-mesothelial mesenchymal cells (Fig. 4l-m). Further, these data suggest that sub-epithelial cells experience higher cortical tension. Altogether, our data show that the two transcriptionally-distinct mesenchymal compartments have different migratory behaviors and mechanical properties.

\section{Sub-mesothelial mesenchymal cells may give rise to vascular smooth muscle}


Our data show that the sub-epithelial mesenchyme gives rise to airway smooth muscle, but computational clustering suggests that the sub-mesothelial mesenchyme is also closely related to smooth muscle. We hypothesized that the sub-mesothelial mesenchyme could give rise to vascular smooth-muscle cells. Mature vascular smooth muscle is not yet present in the lung at $E 11.5^{36}$, but there could be cells in early stages of differentiation down this lineage. We therefore examined the levels of markers enriched in either airway or vascular smooth muscle ${ }^{3}$

(Supplementary Fig. 6a). The expression of airway smooth-muscle genes is highest in the smooth-muscle cluster (Supplementary Fig. 6b-f). Among these, Foxf1, Mylk and Nog are enriched in sub-epithelial compared to sub-mesothelial mesenchyme, as expected given that subepithelial mesenchyme gives rise to airway smooth muscle. Conversely, the vascular smoothmuscle markers Heyl and Speg are slightly enriched in the sub-mesothelial mesenchyme (Supplementary Fig. 6g-h), suggesting that this compartment might contain vascular smoothmuscle precursors. Clustering of smooth-muscle cells alone did not reveal a distinct vascular smooth-muscle population (Supplementary Fig. 6i-p). We therefore examined lungs at E14.5, when vascular smooth muscle is easily detected around blood vessels, and observed that vascular smooth-muscle cells are Lef1 ${ }^{-}$and Foxp $1^{+}$(Supplementary Fig. 6q-s), suggesting that Foxp $1^{+}$, sub-mesothelial mesenchyme may differentiate into vascular smooth muscle.

\section{Diffusion analysis of mesenchymal cells provides insight into differentiation trajectories}

Our scRNA-seq dataset is comprised of undifferentiated mesenchymal cells and mature smooth-muscle cells, and theoretically should include cells at every stage of differentiation between these two states. Live-imaging revealed that smooth-muscle cells originate from the sub-epithelial mesenchyme (Fig. 4). We therefore focused on transitions from the sub-epithelial 
mesenchymal cluster to the smooth-muscle cluster. To search for differentiation trajectories, we implemented diffusion analysis using the Destiny package ${ }^{27}$. Undifferentiated sub-epithelial mesenchymal cells are separated from smooth-muscle cells along diffusion component (DC) 1

(Fig. 5a). The expression of smooth muscle-related genes is positively correlated with DC1, and gene number, a powerful indicator of cell developmental potential ${ }^{37}$, is negatively correlated with DC1 (Fig. 5b-c), suggesting that progression along DC1 represents cells moving away from a stem-cell-like state and differentiating into smooth muscle.

To determine how gene expression evolves over the course of airway smooth-muscle differentiation, we fit the expression of each gene along DC1 and applied unbiased clustering to group genes with similar profiles. We focused on four resultant gene sets that increased along DC1 and one that decreased (Fig. 5d, Supplementary Table 6). The upregulated gene sets (1-4, numbered from latest to earliest) included genes related to smooth muscle and to Wnt, Shh, TGF $\beta$, Bmp, and Hippo signaling pathways, and the downregulated set (5) included the mesenchymal progenitor marker Tbx2 and the distally-expressed ligand Wnt2, suggesting that our analysis had yielded biologically relevant gene sets.

To identify signaling pathways implicated along the smooth-muscle differentiation trajectory, we used KEGG pathway enrichment analysis and carried out motif discovery in promoter and DNase-hypersensitive regions (indicative of accessible chromatin) proximal to genes of each set (Fig. 5e-f, Supplementary Fig. 7). Consistent with our hypothesis that DC1 recapitulated smooth-muscle differentiation, we found that Srf-binding sites (CArG boxes) are highly enriched 
in the promoters and DNase-hypersensitive regions of genes in sets 2 and 3 (Fig. 5f). Further, we found binding sites for Foxf1, a regulator of airway smooth-muscle differentiation ${ }^{38}$, in DNasehypersensitive regions near genes of set 3 (Fig. 5f). We also identified binding sites for the mesenchymal-compartment markers Lef1 and Foxp1 near genes of sets 2, 3, and 4 (Fig. 5f).

All gene sets together, and in particular those that increased in expression along DC1, are enriched for genes involved in Wnt, TGF $\beta$, Hippo, Hedgehog, and MAPK signaling (Fig. 5e). Accordingly, we found motifs for binding sites of Smad-family transcription factors in promoters of genes in set 3, and of Tcf- and Tead-family members in DNase-hypersensitive regions near genes in sets 2-5 (Fig. 5f). Nearly all of these pathways have been implicated in airway smoothmuscle differentiation, with the exception of Hippo signaling. We therefore deleted the Hippo pathway effector Yap1 in the embryonic mesenchyme using Dermo1-Cre and isolated lungs at E12.5. Markers for the sub-epithelial and sub-mesothelial compartments are greatly reduced, as is smooth-muscle wrapping (Fig. 6a), demonstrating that Yap1 is required for patterning and differentiation in the embryonic pulmonary mesenchyme. Our computational analyses can therefore be used to uncover novel signaling pathways important for lung development.

Differentiating mesenchymal cells undergo changes in cell contractility and adhesion to elongate and wrap around the epithelium ${ }^{3}$. Our analyses revealed that genes related to the cytoskeleton, cell adhesion, and the extracellular matrix are upregulated early during differentation, and that their expression accompanies or precedes the expression of the smooth muscle-specific machinery (Acta2) and transcription factors (Myocd) (Fig. 5e, Fig. 6b). These 
data suggest that gene expression in differentiating smooth-muscle cells may be Myocdindependent. In line with this, only a minor fraction (0-6\%) of the smooth-muscle genes identified using diffusion analysis (Fig. 5d) and of smooth-muscle cluster markers (11\%) (Fig. 1d) are differentially expressed in a bulk RNA-seq dataset of E13.5 lungs in which Myocd is deleted from the mesenchyme ${ }^{11}$ (Supplementary Fig. 8). Overall, these data show that the airway smooth muscle gene-expression program is largely Myocd-independent.

Finally, we found that genes that decrease along the smooth-muscle differentiation trajectory are associated with metabolic pathways (Fig. 5e), and that genes involved in proliferative metabolism and biomass production are downregulated along DC1 (Fig. 6c). Indeed, Myc- and Mycn-binding sites are enriched in DNase-hypersensitive regions near genes of set 5 (Fig. 5f), suggesting that proliferativ gene expression is downregulated during differentiation.

Additionally, binding sites for Foxk1 and Foxk2, regulators of glycolysis ${ }^{39}$, are present in the promoters and DNase-hypersensitive regions of gene sets 3 and 4 (Fig. 5f). Overall, our analyses uncover a possible role for metabolic reprogramming during smooth-muscle differentiation.

\section{Wnt signaling activates the early stages of smooth-muscle differentiation to influence epithelial branching}

Our computational analyses suggested that Wnt signaling may be involved in the early stages of airway smooth-muscle differentation. We therefore evaluated the effects of manipulating Wnt signaling on smooth-muscle coverage (percentage of the circumference of the left bronchus wrapped by $\alpha \mathrm{SMA}^{+}$smooth muscle) in LiCl- and IWR1-treated explants. Neither treatment 
signicantly affected smooth-muscle coverage (Fig. 7a-c). Genetic strategies to activate or inhibit Wnt signaling in the pulmonary mesenchyme both lead to disorganized $\alpha \mathrm{SMA}^{+}$smooth-muscle wrapping without overtly changing $\alpha$ SMA levels around the airways ${ }^{17,18}$. Overall, these data suggest that manipulating Wnt signaling has very minor effects on $\alpha$ SMA expression.

This finding was surprising, given the similar epithelial morphologies that result from manipulation of mesenchymal Wnt signaling (Fig. 7d-g) and from manipulation of smoothmuscle differentiation ${ }^{9}$. LiCl-treatment reduces branching frequency and impedes branch extension, similar to lungs with enhanced smooth-muscle differentiation. Conversely, IWR1treated lungs have highly variable branching frequencies, buckled epithelial morphology, and wider branches, much like lungs with reduced smooth-muscle differentiation. Similar to the effects of $\mathrm{LiCl}$ treatment, $A p c$-deletion to enhance Wnt signaling in the mesenchyme leads to constricted buds without increasing aSMA wrapping ${ }^{22}$. Taken together, these data suggest that $\alpha$ SMA-rich smooth muscle is not driving these changes in branching.

Diffusion analyses suggested that differentiating mesenchymal cells could undergo changes in contractility and stiffness quite early, and perhaps before the robust expression of smoothmuscle markers (Fig. 7b). To visualize smooth-muscle cells in the earliest stages of differentiation, we isolated lungs from embryos expressing RFP under the control of the $\alpha$ SMA promoter (SMA-RFP mice). RFP signal can be used to identify nascent smooth-muscle cells that do not have sufficient $\alpha$ SMA for detection by immunofluorescence analysis. Strikingly, we observed a thick layer of F-actin-rich mesenchyme containing many $\mathrm{RFP}^{+}$cells surrounding 
stunted epithelial branches in LiCl-treated explants (Fig. 7h-i). These data suggest that Wnt activates the earliest stages of smooth-muscle differentiation. Consistently, genetically activating mesenchymal Wnt signaling leads to widespread expression of $B m p 4$, one of the earliest genes activated along the smooth-muscle differentiation trajectory, and dense mesenchymal-cell packing around the epithelium, preventing branching ${ }^{22}$. Here, we observed that local accumulation of F-actin, indicative of mesenchymal stiffening ${ }^{40,41}$, similarly prevented epithelial branch outgrowth.

\section{Discussion}

Here, we provide the first single-cell overview of cell identities and transitions in the embryonic pulmonary mesenchyme during branching morphogenesis of the mouse lung. scRNAseq analyses revealed compartment-specific transcriptional heterogeneity across the mesenchyme and uncovered how signaling pathways evolve over the course of airway smooth-muscle differentiation. Building off of our computational findings, we identify Wnt signaling as a master regulator of mesenchymal patterning in early lung development and show that Wnt-dependent activation of smooth-muscle differentiation and mesenchymal stiffening can physically influence airway epithelial branching.

Our data show that the pulmonary mesenchyme contains a continuum of cell identites, which may explain why a distinct smooth-muscle progenitor marker has remained elusive ${ }^{25}$. Unbiased lineage tracing in fixed samples previously showed that new smooth-muscle cells at branch tips are members of clones from the adjacent sub-epithelial mesenchyme ${ }^{18}$. Our live-imaging data 
support these findings, and also reveal a surprising level of cell motility within the embryonic pulmonary mesenchyme. Similar dynamic behaviors have been observed in kidney development $^{42}$. The extent and timescales of these rearrangements raise questions about how focal morphogen sources proposed to act as chemoattractants could be maintained as mesenchymal cells migrate during branching morphogenesis ${ }^{43,44}$.

The heterogeneity of mesenchymal cells within developing tissues influences how they respond to morphogenetic signals ${ }^{45}$ and can define if and when they differentiate. Exposing transplanted stalk mesenchyme to a Wnt signal primes it to migrate into a donor tissue and form smooth muscle ${ }^{18}$. It is possible that stalk mesenchyme is comprised of sub-mesothelial mesenchymal cells, and that activation of Wnt signaling converts them to a sub-epithelial identity, which we have shown here is associated with greater cytoskeletal tension and motility.

We and others have shown that Fgf9/Shh ${ }^{16}$ and Wnt signaling spanning the epithelium to the mesothelium patterns the embryonic pulmonary mesenchyme. Epithelium-derived Wnt signals also radially pattern smooth-muscle differentiation in the ureteric mesenchyme ${ }^{46}$. In the chick intestine, opposing molecular signals from the epithelium and the mesothelium, as well as mechanical forces generated by growth of the intestinal epithelium and contraction of smooth muscle, are integrated to spatiotemporally pattern the differentiation of multiple smooth-muscle layers $^{47}$. The role of mechanical forces in regulating pulomonary mesenchymal cell identity have yet to be fully elucidated. 
Reconstructing the smooth-muscle differentiation trajectory revealed that genes related to the cytoskeleton and cell-matrix adhesions are expressed early during differentiation. These changes could lead to tissue stiffening, which regulates diverse morphogenetic events ${ }^{41,48,49}$. Indeed, we found that Wnt-dependent accumulation of nascent smooth-muscle cells and F-actin around emerging branches is associated with local inhibition of epithelial growth. Similarly, activating Wnt signaling in the ureteric mesenchyme leads to ectopic accumulation of smooth-muscle progenitors and a hypoplastic ureter ${ }^{46}$. F-actin levels can reflect cell and extracellular matrix stiffening ${ }^{40,41}$, and Wnt signaling can activate actomyosin contractility ${ }^{50}$ and matrix deposition ${ }^{19}$, possibly contributing to tissue stiffening around emerging branches. VinTS experiments revealed higher cortical tension in the sub-epithelial mesenchyme, which may allow it to physically sculpt the branching epithelium. Activation of Wnt signaling to promote sub-epithelial mesenchymal fates could enhance this effect.

Our results raise questions about which stage(s) of smooth-muscle differentiation and which aspects of smooth-muscle cell identity influence branching morphogenesis. The smooth-muscle gene-expression program extends beyond the influence of a single transcription factor (Myocd), suggesting that developing smooth muscle may be robust to the loss of single components. Indeed, loss of Acta2 is compensated for by other actin isoforms ${ }^{12,13}$. In the developing lung, Myocd-deletion decreases the expression of only a few smooth-muscle genes, and in the developing ureter, expansion of smooth-muscle progenitors occurs with minimal increase in the expression of Myocd $^{46}$. Consistently, Myocd expression is insufficient to drive the entire smoothmuscle gene-expression program in cell culture ${ }^{51}$. 
Our scRNA-seq analyses have uncovered novel regulators of mesenchymal patterning during lung development. We computationally identified and genetically validated a role for Hippo signaling via Yap1 in the embryonic pulmonary mesenchyme. Mesenchymal Yap regulates smooth-muscle differentiation around the gastrointestinal epithelium ${ }^{52}$, and crosstalk between Wnt and Yap signaling regulates intestinal stem cell homeostasis ${ }^{53}$. Future investigations will elucidate how Yap influences airway smooth-muscle differentiation and whether cooperation with Wnt signaling is involved. Our data also reveal that sub-epithelial cells systematically downregulate enzymes required for proliferative metabolism as they differentiate into smooth muscle, and that Foxk1/2, regulators of glycolysis ${ }^{39}$, may be involved in differentiation. Metabolic reprogramming is a critical component of stem cell maintenance and differentiation ${ }^{54}$, and enhanced glycolysis acts via Yap to regulate neural crest migration ${ }^{55}$. Notably, Foxk1 interacts with Srf to repress expression of genes including $A c t a 2^{56}$, suggesting that metabolic reprogramming participates in airway smooth-muscle differentiation.

In conclusion, our findings reveal the heterogeneity of the embryonic pulmonary mesenchyme and the multitude of signals involved in smooth-muscle differentiation. Our computational analyses lay the foundation for future investigations into the mechanisms that regulate cell identity and differentiation in the pulmonary mesenchyme. Further, our approaches could be extended to other organs or organisms to compare tissue patterning and differentiation in diverse developmental contexts. 


\section{Online Methods}

\section{Mice}

Breeding of CD1, Dermo1-Cre (JAX 008712), R26R-Confetti (JAX 013731), mTmG (JAX 007676), SMA-RFP, and Yap-flox (JAX 027929) mice and isolation of embryos were carried out in accordance with institutional guidelines following the NIH Guide for the Care and Use of Laboratory Animals and approved by Princeton's Institutional Animal Care and Use Committees. Breeding of VinTS mice ${ }^{35}$ and isolation of embryos were carried out in accordance with the Animals for Research Act of Ontario and the Guidelines of the Canadian Council on Animal Care, and procedures were approved by the Hospital for Sick Children Animal Care Committee. Dermo1-Cre/+;Confetti/+ embryos were obtained by mating Confetti homozygous females to Dermo1-Cre heterozygous males. Yap-flox and mTmG mice were bred to generate homozygous Yap-flox;mTmG mice. Dermo1-Cre males were bred to Yap-flox females to generate Dermo1-Cre/+;Yap-fl/+ males, and these were then mated to Yap-flox;mTmG females to generate Dermo1-Cre/+;Yap-fl/fl;mTmG/+ and control Dermo1-Cre/+;Yap-fl/+;mTmG/+ littermates. Pups and embryos from were genotyped for Cre, mTmG, and/or Yap-flox by isolating DNA from the tail snips (pups) or from the head of each embryo, followed by PCR and gel electrophoresis. The forward primer sequence for Cre was

GCATTACCGGTCGATGCAACGAGTGATGAG and the reverse primer sequence was GAGTGAACGAACCTGGTCGAAATCAGTGCG. The primer sequences for Yap-flox and $\mathrm{mTmG}$ are provided in the Jackson Labs genotyping protocol for each strain. Where possible, mGFP expression from mTmG was used for genotyping.

\section{scRNA-seq experiments}


Lungs were dissected from CD1 mouse embryos collected at E11.5 in cold PBS. Isolated left lobes were grouped by stage, placed in dispase, and mechanically dissociated with tungsten needles. After 10 minutes in dispase at room temperature, DMEM without HEPES and supplemented with 5\% fetal bovine serum (FBS, Atlanta Biologicals) was added, and cell suspensions were passed through a filter with $40-\mu \mathrm{m}$-diameter pores. The resultant cell suspensions were then processed by the Princeton Genomics Core Facility. The two sample groups were processed separately. Cells were loaded and processed using the Chromium Single Cell 3' Library and Gel Bead Kit v2 on the Chromium Controller (10X Genomics) following manufacturer protocols. Individual cells were encapsulated in droplets with single gel beads carrying unique barcoded primers and then lysed. Then cDNA fragments were synthesized, barcoded, and amplified by PCR. Illumina sequencing libraries were prepared from the amplified cDNA from each sample group using the Nextra DNA library prep kit (Illumina). The v2 libraries were sequenced on Illumina HiSeq 2500 Rapid flowcells (Illumina) as paired-end $26+$ 50 nucleotide reads following manufacturer protocols. Base calling was performed, and raw sequencing reads were filtered using the Illumina sequencer control software to keep only passfiltered reads for downstream analysis. The 10x CellRanger software version 2.0.1 was used to run the count pipeline with default settings on all FASTQ files from each sample to generate gene-barcode matrices using the Mus musculus reference genome mm10-1.2.0.

\section{scRNA-seq data analysis}

scRNA-seq data exported from Cell Ranger were imported into R and processed using the Seurat package ${ }^{28}$. Datasets from all groups were normalized and then integrated based on 2000 variable features (genes) identified by the Seurat function FindVariableFeatures. The 
integrated dataset was then scaled and analyzed to find neighbors and clusters with the Seurat functions FindNeighbors and FindClusters. Finally, the uniform manifold approximation and projection (UMAP) dimensional reduction was performed to visualize clusters. For initial analyses, all cells were used, which revealed large clusters of blood and immune cells (Supplementary Fig. 1k-l). Since these were not the cell populations of interest, we removed them from further analysis (Supplementary Fig. 1m-n). We then adjusted the dataset to account for cell-to-cell variability based on cell-cycle stage using the Seurat package. The resulting filtered and adjusted dataset was used for all subsequent analyses. Cluster identities were then assigned based on markers enriched in each cluster that are known to be enriched in each lung cell population. To investigate mesenchymal cells specifically, we repeated the Seurat pipeline outlined above including only cells from clusters 0, 1, and 2 (Supplementary Fig. 2). We generated a UMAP, identified markers for each of the 5 new clusters identified, and examined the expression of putative progenitor markers in each cluster (Supplementary Fig. 2).

Using published in situ hybridization results available on MGI, we compiled a list of genes that have been detected in the pulmonary mesenchyme at $E 11.5-E 12.5$. Based on MGI entries containing images, we classified the expression pattern of each gene as either subepithelial, sub-mesothelial, smooth muscle, everywhere, or weak (Supplementary Table 3). Genes that did not fit any of these broad categories because of even more limited expression domains were excluded. A subset of the genes classified as sub-epithelial or sub-mesothelial were detected and included as variable features by the Seurat algorithm. Using the heatmap.2 function in $\mathrm{R}$, we clustered sub-epithelial and sub-mesothelial cells based on their expression of 
this subset of genes (columns in Fig. 2e) and clustered the genes from the curated MGI list (rows in Fig. 2e).

To search for functional differences between the mesenchymal clusters identified in Fig. 1, we identified genes that were differentially expressed in cluster 0 compared to cluster 1 using the FindMarkers function from the Seurat package, and then grouped these based on whether they were upregulated in cluster 0 or in cluster 1 . These gene lists were then subjected to GO enrichment analysis using the clusterProfiler package in $\mathrm{R}^{57}$.

\section{Diffusion analysis of mesenchymal cells}

To reconstruct smooth-muscle differentiation trajectories, we used the cell-by-gene matrix of 2000 variable genes with scaled expression values generated using the Seurat algorithm and applied the Destiny package in $\mathrm{R}^{27}$. Only clusters 0 and 2 were used in this analysis. Three cells from cluster 0 appeared as clear outliers and were excluded from this analysis. To search for groups of genes with similar expression patterns over the course of smooth-muscle differentiation, we first used the edge R package in $\mathrm{R}$ to fit the expression of all genes along DC1 using a spline model with three degrees of freedom ${ }^{58}$. The resulting fits were then filtered by adjusted $p$ value $<0.05$ obtained by implementing a generalized likelihood ratio test with the lrt function from the edgeR package ${ }^{58}$. These fits were then clustered using hierarchical agglomerative clustering with average linkage based on Pearson correlation distance to identify gene sets with similar expression profiles along DC1. Gene sets of interest were then subjected to KEGG pathway enrichment analysis implemented using clusterProfiler ${ }^{57}$. 


\section{Motif discovery}

Motif discovery was carried out by examining promoter regions and DNasehypersensitive regions near genes of interest using a custom Python pipeline and HOMER ${ }^{59}$. Genomic regions based on the mouse ENCODE E14 lung DNase-hypersensitivity dataset ${ }^{60}$ were $^{-1}$ matched to genes from the gene sets identified in Fig. 5d based on proximity to their transcriptional start site. The sequences of these DNase-hypersensitive regions were then extracted and analyzed using HOMER findMotifs.pl FASTA motif analysis tool ${ }^{59}$ to identify overrepresented motifs compared to a background file of DNase-hypersensitive regions located near the transcriptional start sites of a list of genes randomly generated with the Regulatory Sequence Analysis Tools (RSAT) web interface ${ }^{61}$.

\section{Organ explant culture and live imaging}

Lungs from E11.5 CD1 mice were dissected in cold, sterile PBS supplemented with antibiotics (50 units/ml of penicillin and streptomycin) and then cultured on porous membranes (nucleopore polycarbonate track-etch membrane, $8 \mu \mathrm{m}$ pore size, $25 \mathrm{~mm}$ diameter; Whatman) floating on top of DMEM/F12 medium (without HEPES) supplemented with 5\% FBS and antibiotics (50 units/ml of penicillin and streptomycin) for $24 \mathrm{hr}$. Reagents used to manipulate Wnt signaling included LiCl (10 mM; Sigma) and IWR1 (100 $\mu \mathrm{M}$; Sigma). To inhibit Shh signaling we used cyclopamine ( $1 \mu \mathrm{M}$; Tocris). For live-imaging analysis, Dermo1Cre/+;Confetti/+ lungs were cultured on Transwell filters (polyethylene terephthalate membrane, $3 \mu \mathrm{m}$ pore size, $10.5 \mathrm{~mm}$ diameter; Corning) within a stage-top incubator (Pathology Devices). Frames were acquired every 30 or $60 \mathrm{~min}$ for up to $48 \mathrm{hr}$ under brightfield (1-2 ms exposure per 
plane for a total of seven planes per time point) or spinning disk confocal illumination (X-light, $122 \mathrm{~ms}$ exposure per plane for 5-7 planes per time point) on an inverted microscope (Nikon Ti).

\section{Vinculin tension sensor experiments}

Lungs were isolated from E12.5 VinTS embryos and embedded in $1 \%$ low melting point agarose on a coverslip fitted into a custom imaging chamber. After an hour of incubation at $37^{\circ} \mathrm{C}$, cell culture medium supplemented with $5 \%$ FBS was added to the chamber and samples were imaged by confocal and fluorescence lifetime microscopy (FLIM). Imaging and analyses were performed as previously described ${ }^{35}$. Briefly, FLIM was carried out on a Nikon A1R Si laser-scanning confocal microscope with a PicoHarp 300 TCSPC module and a 440 nm pulseddiode laser (Picoquant). Imaging was performed with a 40×/1.25 NA water immersion objective. For each lung, 2 to $3 \mathrm{z}$-stacks at different locations along the distal left lobe were acquired. Fluorescence lifetime of the donor fluorophore (mTFP1) was estimated using FLIM Fit 5.1.1 ${ }^{62}$. Cells of interest were segmented manually in FLIM Fit to obtain an average lifetime per cell. Sub-epithelial mesenchymal cells were at least 2-cell bodies away the from the mesothelium, and sub-mesothelial mesenchymal cells were within 2-cell bodies of the mesothelium.

\section{Tissue sectioning, immunofluorescence analysis, and imaging}

Isolated lungs were fixed in 4\% paraformaldehyde in PBS for 15 minutes at room temperature. For sectioning, lungs were washed first in $20 \%$ sucrose in PBS, then $30 \%$ sucrose in PBS, then left overnight in a 1:1 mixture of OCT and 30\% sucrose prior to embedding and freezing in OCT. A Leica CM3050S cryostat was then used to create $10-\mu \mathrm{m}$-thick sections for 
staining on slides. For whole-mount staining and for sections on slides, samples were washed with $0.1 \%$ Triton X-100 in PBS and then blocked with 5\% goat serum and 0.1\% BSA. Samples were then incubated with primary antibodies against $\alpha$ SMA (Sigma a5228, 1:400), Axin2 (Abcam ab32197, 1:200), $\beta$-catenin (Sigma SAB4500541, 1:200), E-cadherin (Cell Signaling 3195, 1:200 or Invitrogen 13-1900, 1:200), Foxp1 (Cell Signaling 2005, 1:200), GFP (Invitrogen A-11122, 1:500), Lef1 (Cell Signaling 2230, 1:200), PECAM (Abcam ab28364, 1:200), or RFP (Abcam ab62341, 1:400), followed by incubation with Alexa Fluor-conjugated secondary antibodies (1:200), Alexa Fluor-conjugated phalloidin (1:500) and/or Hoechst (1:1000). Sections on slides were then mounted in Fluorosave. Whole lungs were dehydrated in a methanol series and cleared with Murray's clear (1:2 ratio of benzyl alcohol to benzyl benzoate). To preserve endogenous fluorescence of Confetti and VinTS lungs, samples were not dehydrated or cleared, but instead mounted in Fluorosave. Confocal stacks were collected using a spinning disk confocal (BioVision X-Light V2) on an inverted microscope (Nikon Ti) using either 20× air, 40× oil, or 60× oil objectives (Nikon).

\section{Image analysis and statistics}

We developed a simple pipeline in MatLab to quantify fluorescence intensity profiles. First, images of Lef1 or Foxp1 immunostaining were background subtracted and then lines were traced either starting at the edge of branch L.L2 and moving outward into the mesenchyme for Lef1 or starting at the mesothelium near branch L.L2 and moving inward into the mesenchyme for Foxp1. Average fluorescence intensity in a $16 \times 16$ pixel window $(5.7 \times 5.7 \mu \mathrm{m})$ was measured at each point along the line, and the calculation included only the intensity of pixels within cell nuclei based on a mask generated from Hoechst staining. Intensity profiles were 
averaged from 5 distinct lines per sample, and the resultant curves were compared using twoway ANOVA in GraphPad Prism 5. A similar pipeline was used to quantify Axin2, $\beta$-catenin, and F-actin intensity, with small modifications. Axin2 intensity was only averaged from regions with no Hoechst signal, and $\beta$-catenin levels were compared as the ratio of mean nuclear $\left(\right.$ Hoechst $\left.^{+}\right)$to mean cytoplasmic $\left(\right.$Hoechst $\left.^{-}\right)$pixels. F-actin intensity was measured from all pixels (no mask).

Smooth-muscle coverage was quantified as described previously ${ }^{9}$. Briefly, the outline of the epithelium was traced in reconstructed cross-sections through the left primary bronchus at 5 different planes, and $\alpha$ SMA fluorescence intensity was measured in a $16 \times 16$ pixel $(5.7 \times 5.7 \mu \mathrm{m})$ window at each point along the outline. Percent coverage was defined as the percentage of the epithelial outline with above-threshold aSMA staining intensity. Sample groups were compared using a two-sided t-test in GraphPad Prism 5.

\section{Time-lapse analysis}

Cells from time-lapses of Dermo1-Cre/+;Confetti/+ embryos were tracked manually in Fiji for as many consecutive time points as possible, and the resultant tracks were analyzed in MatLab. To estimate cell speeds, we first corrected cell tracks for local tissue movement and drift. Since the direction and magnitude of lung growth depends on location within the organ, we corrected cell tracks based on local displacements: for each cell, we computed the mean displacement of all cells within a 150 pixel $(111 \mu \mathrm{m})$ radius as an estimate of local tissue movement, and then subtracted the cumulative sum of these displacements from the original cell's track at each time point. This approach allowed us to independently correct for proximal- 
distal movements near the elongating primary bronchus and lateral movements near expanding branch tips (see tracks for smooth-muscle cells vs. sub-epithelial and sub-mesothelial mesenchymal cells in Supplementary Fig. 5d). Corrected tracks had slightly smaller instantaneous speeds and much smaller overall displacements (Supplementary Fig. 5e-f), indicating that our approach had removed the effects of drift and tissue growth. MSD curves for each tracked cell were generated based on time intervals of up to 7 hours and fit with a $1^{\text {st }}$ degree polynomial to estimate persistence. Only fits with $\mathrm{R}^{2}$ greater than 0.4 were included in the comparison between samples. To generate the pie charts in Fig. 4, cells were assigned to a tissue compartment (sub-epithelial mesenchyme, sub-mesothelial mesenchyme, or smooth muscle) based on their location at the start of their track, and classified as staying within their compartment or crossing over by comparing the entire track to tissue growth in the brightfield channel. The boundary between sub-epithelial and sub-mesothelial mesenchyme was between 2and 3-cell bodies away from the mesothelium. Cells were classified as differentiating into smooth muscle based on their positions and their progressively elongating morphology.

\section{Analysis of bulk RNA-seq of Myocd-mutant lungs}

Bulk RNA-seq data for 5 control and 5 mutant E13.5 lungs in which Myocd had been deleted from the mesenchyme were downloaded from GEO (accession number GSE143394 ${ }^{11}$ ) and processed using the DESeq2 package ${ }^{63}$. Briefly, the DESeq2 package was used to import data, estimate size factors and dispersions, and then calculate differential expression of genes and significance using the Wald test. Log fold changes were shrunk according to the DESeq2 package guidelines. We then used volcano plots to visualize adjusted $p$-values and $\log _{2}$ fold changes for either genes that correlated positively (sets 1-4) or negatively (set 5) with DC1 in 
Fig. 5d (Supplementary Fig. 8a) or smooth-muscle cluster marker genes from Fig. 1b, d (Supplementary Fig. 8c). Since most of the genes were not significantly differentially expressed, we also plotted adjusted $p$-values against the mean of regularized log transformed counts obtained using the DESeq2 package to confirm that these genes were detected at high enough levels in the bulk RNA-seq dataset and found that there was no bias of differential expression based on transcript abundance (Supplementary Fig. 8b, d).

\section{Acknowledgements}

We would like to acknowledge Dr. Wei Wang and the Genomics Core Facility of Princeton University. We would also like to thank members of the Tissue Morphodynamics Group for helpful discussions and feedback on the mnauscript. This work was supported by an NIH/NICHD R01 (HD0990300), an HHMI Faculty Scholars Award, and an NIH/NHLBI R01 (HL120142) to C.M.N and a CIHR award (MOP 126115) to S.H. K.G. was supported in part by a postgraduate scholarship-doctoral (PGS-D) from the Natural Sciences and Engineering Research Council of Canada and the Dr. Margaret McWilliams Predoctoral Fellowship from the Canadian Federation of University Women. J.M.J. was supported in part by an NIH NRSA Fellowship (F30 HL139039).

\section{Author Contributions}

K.G. and C.M.N. conceptualized the study, designed the experiments, intepreted the data, and wrote the manuscript. K.G. performed the experiments and collected the data. J.M.J. carried 
out the motif discovery analysis. H.T., M.Z., and S.H. provided all reagents and technical assistance for obtaining VinTS results. All authors provided input on the final manuscript.

\section{Competing interests}

The authors declare no competing interests.

\section{References}

1 Shyer, A. E. et al. Villification: how the gut gets its villi. Science 342, 212-218, doi:10.1126/science.1238842 (2013).

2 Neumann, N. M. et al. Coordination of Receptor Tyrosine Kinase Signaling and Interfacial Tension Dynamics Drives Radial Intercalation and Tube Elongation. Dev Cell 45, 67 82 e66, doi:10.1016/j.devcel.2018.03.011 (2018).

3 Jaslove, J. M. \& Nelson, C. M. Smooth muscle: a stiff sculptor of epithelial shapes. Philos Trans R Soc Lond B Biol Sci 373, doi:10.1098/rstb.2017.0318 (2018).

4 Roman, J., Schuyler, W., McDonald, J. A. \& Roser, S. Heparin inhibits lung branching morphogenesis: potential role of smooth muscle cells in cleft formation. Am J Med Sci 316, 368378, doi:10.1097/00000441-199812000-00003 (1998).

5 Yamada, T., Suzuki, E., Gejyo, F. \& Ushiki, T. Developmental changes in the structure of the rat fetal lung, with special reference to the airway smooth muscle and vasculature. Arch Histol Cytol 65, 55-69, doi:10.1679/aohc.65.55 (2002).

6 Leslie, K. O., Mitchell, J. J., Woodcock-Mitchell, J. L. \& Low, R. B. Alpha smooth muscle actin expression in developing and adult human lung. Differentiation 44, 143-149, doi:10.1111/j.1432-0436.1990.tb00547.x (1990).

7 Kim, N. \& Vu, T. H. Parabronchial smooth muscle cells and alveolar myofibroblasts in lung development. Birth Defects Res C Embryo Today 78, 80-89, doi:10.1002/bdrc.20062 (2006).

8 Danopoulos, S. et al. Human lung branching morphogenesis is orchestrated by the spatiotemporal distribution of ACTA2, SOX2, and SOX9. Am J Physiol Lung Cell Mol Physiol 314, L144-L149, doi:10.1152/ajplung.00379.2017 (2018).

9 Goodwin, K. et al. Smooth muscle differentiation shapes domain branches during mouse lung development. Development, doi:10.1242/dev.181172 (2019). 
10 Kim, H. Y. et al. Localized Smooth Muscle Differentiation Is Essential for Epithelial Bifurcation during Branching Morphogenesis of the Mammalian Lung. Dev Cell 34, 719-726, doi:10.1016/j.devcel.2015.08.012 (2015).

11 Young, R. E. et al. Smooth Muscle Differentiation Is Essential for Airway Size, Tracheal Cartilage Segmentation, but Dispensable for Epithelial Branching. Dev Cell, doi:10.1016/j.devcel.2020.02.001 (2020).

12 Schildmeyer, L. A. et al. Impaired vascular contractility and blood pressure homeostasis in the smooth muscle alpha-actin null mouse. FASEB J 14, 2213-2220, doi:10.1096/fj.990927com (2000).

13 Haaksma, C. J., Schwartz, R. J. \& Tomasek, J. J. Myoepithelial cell contraction and milk ejection are impaired in mammary glands of mice lacking smooth muscle alpha-actin. Biol Reprod 85, 13-21, doi:10.1095/biolreprod.110.090639 (2011).

14 Miller, L. A. et al. Role of Sonic hedgehog in patterning of tracheal-bronchial cartilage and the peripheral lung. Dev Dyn 231, 57-71, doi:10.1002/dvdy.20105 (2004).

15 El Agha, E., Kheirollahi, V., Moiseenko, A., Seeger, W. \& Bellusci, S. Ex vivo analysis of the contribution of FGF10(+) cells to airway smooth muscle cell formation during early lung development. Dev Dyn 246, 531-538, doi:10.1002/dvdy.24504 (2017).

16 White, A. C. et al. FGF9 and SHH signaling coordinate lung growth and development through regulation of distinct mesenchymal domains. Development 133, 1507-1517, doi:10.1242/dev.02313 (2006).

17 De Langhe, S. P. et al. Formation and differentiation of multiple mesenchymal lineages during lung development is regulated by beta-catenin signaling. PLoS One 3, e1516, doi:10.1371/journal.pone.0001516 (2008).

18 Kumar, M. E. et al. Mesenchymal cells. Defining a mesenchymal progenitor niche at single-cell resolution. Science 346, 1258810, doi:10.1126/science.1258810 (2014).

19 De Langhe, S. P. et al. Dickkopf-1 (DKK1) reveals that fibronectin is a major target of Wnt signaling in branching morphogenesis of the mouse embryonic lung. Dev Biol 277, 316331, doi:10.1016/j.ydbio.2004.09.023 (2005).

20 Ludtke, T. H. et al. Tbx2 and Tbx3 Act Downstream of Shh to Maintain Canonical Wnt Signaling during Branching Morphogenesis of the Murine Lung. Dev Cell 39, 239-253, doi:10.1016/j.devcel.2016.08.007 (2016).

21 Yin, Y. et al. An FGF-WNT gene regulatory network controls lung mesenchyme development. Dev Biol 319, 426-436, doi:10.1016/j.ydbio.2008.04.009 (2008).

22 Luo, Y. et al. Mesenchymal adenomatous polyposis coli plays critical and diverse roles in regulating lung development. BMC Biol 13, 42, doi:10.1186/s12915-015-0153-1 (2015).

23 Goss, A. M. et al. Wnt2 signaling is necessary and sufficient to activate the airway smooth muscle program in the lung by regulating myocardin/Mrtf-B and Fgf10 expression. Dev Biol 356, 541-552, doi:10.1016/j.ydbio.2011.06.011 (2011). 
24 Mailleux, A. A. et al. Fgf10 expression identifies parabronchial smooth muscle cell progenitors and is required for their entry into the smooth muscle cell lineage. Development 132, 2157-2166, doi:10.1242/dev.01795 (2005).

25 Moiseenko, A. et al. Origin and characterization of alpha smooth muscle actin-positive cells during murine lung development. Stem Cells 35, 1566-1578, doi:10.1002/stem.2615 (2017).

26 Tritschler, S. et al. Concepts and limitations for learning developmental trajectories from single cell genomics. Development 146, doi:10.1242/dev.170506 (2019).

27 Haghverdi, L., Buettner, F. \& Theis, F. J. Diffusion maps for high-dimensional singlecell analysis of differentiation data. Bioinformatics 31, 2989-2998, doi:10.1093/bioinformatics/btv325 (2015).

28 Butler, A., Hoffman, P., Smibert, P., Papalexi, E. \& Satija, R. Integrating single-cell transcriptomic data across different conditions, technologies, and species. Nat Biotechnol 36, 411-420, doi:10.1038/nbt.4096 (2018).

29 Giraddi, R. R. et al. Single-Cell Transcriptomes Distinguish Stem Cell State Changes and Lineage Specification Programs in Early Mammary Gland Development. Cell Rep 24, 16531666 e1657, doi:10.1016/j.celrep.2018.07.025 (2018).

30 Brunskill, E. W. et al. Single cell dissection of early kidney development: multilineage priming. Development 141, 3093-3101, doi:10.1242/dev.110601 (2014).

31 Eastman, Q. \& Grosschedl, R. Regulation of LEF-1/TCF transcription factors by Wnt and other signals. Curr Opin Cell Biol 11, 233-240 (1999).

32 Al Alam, D. et al. Contrasting expression of canonical Wnt signaling reporters TOPGAL, BATGAL and Axin2(LacZ) during murine lung development and repair. PLoS One 6, e23139, doi:10.1371/journal.pone.0023139 (2011).

33 Metzger, R. J., Klein, O. D., Martin, G. R. \& Krasnow, M. A. The branching programme of mouse lung development. Nature 453, 745-750, doi:10.1038/nature07005 (2008).

34 Grashoff, C. et al. Measuring mechanical tension across vinculin reveals regulation of focal adhesion dynamics. Nature 466, 263-266, doi:10.1038/nature09198 (2010).

35 Tao, H. et al. Oscillatory cortical forces promote three dimensional cell intercalations that shape the murine mandibular arch. Nat Commun 10, 1703, doi:10.1038/s41467-019-09540-z (2019).

36 Badri, K. R., Zhou, Y. \& Schuger, L. Embryological origin of airway smooth muscle. Proc Am Thorac Soc 5, 4-10, doi:10.1513/pats.200704-049VS (2008).

37 Gulati, G. S. et al. Single-cell transcriptional diversity is a hallmark of developmental potential. Science 367, 405-411, doi:10.1126/science.aax0249 (2020).

38 Ustiyan, V. et al. FOXF1 transcription factor promotes lung morphogenesis by inducing cellular proliferation in fetal lung mesenchyme. Dev Biol 443, 50-63, doi:10.1016/j.ydbio.2018.08.011 (2018). 
39 Sukonina, V. et al. FOXK1 and FOXK2 regulate aerobic glycolysis. Nature 566, 279283, doi:10.1038/s41586-019-0900-5 (2019).

40 Solon, J., Levental, I., Sengupta, K., Georges, P. C. \& Janmey, P. A. Fibroblast adaptation and stiffness matching to soft elastic substrates. Biophys J 93, 4453-4461, doi:10.1529/biophysj.106.101386 (2007).

41 Heer, N. C. \& Martin, A. C. Tension, contraction and tissue morphogenesis. Development 144, 4249-4260, doi:10.1242/dev.151282 (2017).

42 Combes, A. N., Lefevre, J. G., Wilson, S., Hamilton, N. A. \& Little, M. H. Cap mesenchyme cell swarming during kidney development is influenced by attraction, repulsion, and adhesion to the ureteric tip. Dev Biol 418, 297-306, doi:10.1016/j.ydbio.2016.06.028 (2016).

43 Menshykau, D., Blanc, P., Unal, E., Sapin, V. \& Iber, D. An interplay of geometry and signaling enables robust lung branching morphogenesis. Development 141, 4526-4536, doi:10.1242/dev.116202 (2014).

44 Metzger, R. J. \& Krasnow, M. A. Genetic control of branching morphogenesis. Science 284, 1635-1639, doi:10.1126/science.284.5420.1635 (1999).

45 Reinhardt, R. et al. Molecular signatures identify immature mesenchymal progenitors in early mouse limb buds that respond differentially to morphogen signaling. Development 146, doi:10.1242/dev.173328 (2019).

46 Trowe, M. O. et al. Canonical Wnt signaling regulates smooth muscle precursor development in the mouse ureter. Development 139, 3099-3108, doi:10.1242/dev.077388 (2012).

47 Huycke, T. R. et al. Genetic and Mechanical Regulation of Intestinal Smooth Muscle Development. Cell 179, 90-105 e121, doi:10.1016/j.cell.2019.08.041 (2019).

48 Barriga, E. H., Franze, K., Charras, G. \& Mayor, R. Tissue stiffening coordinates morphogenesis by triggering collective cell migration in vivo. Nature 554, 523-527, doi:10.1038/nature25742 (2018).

49 Zhu, M. et al. Spatial mapping of tissue properties in vivo reveals a 3D stiffness gradient in the mouse limb bud. Proc Natl Acad Sci U S A 117, 4781-4791, doi:10.1073/pnas.1912656117 (2020).

50 Lee, J. Y. et al. Wnt/Frizzled signaling controls C. elegans gastrulation by activating actomyosin contractility. Curr Biol 16, 1986-1997, doi:10.1016/j.cub.2006.08.090 (2006).

51 Yoshida, T., Kawai-Kowase, K. \& Owens, G. K. Forced expression of myocardin is not sufficient for induction of smooth muscle differentiation in multipotential embryonic cells. Arterioscler Thromb Vasc Biol 24, 1596-1601, doi:10.1161/01.ATV.0000137190.63214.c5 (2004).

52 Cotton, J. L. et al. YAP/TAZ and Hedgehog Coordinate Growth and Patterning in Gastrointestinal Mesenchyme. Dev Cell 43, 35-47 e34, doi:10.1016/j.devcel.2017.08.019 (2017). 
53 Li, Q. et al. Lats1/2 Sustain Intestinal Stem Cells and Wnt Activation through TEADDependent and Independent Transcription. Cell Stem Cell, doi:10.1016/j.stem.2020.03.002 (2020).

54 Intlekofer, A. M. \& Finley, L. W. S. Metabolic signatures of cancer cells and stem cells. Nat Metab 1, 177-188, doi:10.1038/s42255-019-0032-0 (2019).

55 Bhattacharya, D., Azambuja, A. P. \& Simoes-Costa, M. Metabolic Reprogramming Promotes Neural Crest Migration via Yap/Tead Signaling. Dev Cell 53, 199-211 e196, doi:10.1016/j.devcel.2020.03.005 (2020).

56 Freddie, C. T., Ji, Z., Marais, A. \& Sharrocks, A. D. Functional interactions between the Forkhead transcription factor FOXK1 and the MADS-box protein SRF. Nucleic Acids Res 35, 5203-5212, doi:10.1093/nar/gkm528 (2007).

57 Yu, G., Wang, L. G., Han, Y. \& He, Q. Y. clusterProfiler: an R package for comparing biological themes among gene clusters. OMICS 16, 284-287, doi:10.1089/omi.2011.0118 (2012).

58 Storey, J. D. L., J. T.; Bass, A. J. edge: Extraction of Differential Gene Expression. $R$ package version 2.16.0 (2019).

59 Heinz, S. et al. Simple combinations of lineage-determining transcription factors prime cis-regulatory elements required for macrophage and B cell identities. Mol Cell 38, 576-589, doi:10.1016/j.molcel.2010.05.004 (2010).

60 Yue, F. et al. A comparative encyclopedia of DNA elements in the mouse genome.

Nature 515, 355-364, doi:10.1038/nature13992 (2014).

61 Nguyen, N. T. T. et al. RSAT 2018: regulatory sequence analysis tools 20th anniversary. Nucleic Acids Res 46, W209-W214, doi:10.1093/nar/gky317 (2018).

62 Warren, S. C. et al. Rapid global fitting of large fluorescence lifetime imaging microscopy datasets. PLoS One 8, e70687, doi:10.1371/journal.pone.0070687 (2013).

63 Love, M. I., Huber, W. \& Anders, S. Moderated estimation of fold change and dispersion for RNA-seq data with DESeq2. Genome Biol 15, 550, doi:10.1186/s13059-014-0550-8 (2014). 


\section{Figure legends}

\section{Figure 1. Clustering scRNA-seq data from $E 11.5$ mouse lungs reveals expected cell}

populations. (a) Image of sample lung used for scRNA-seq experiment. Each lung was microdissected to isolate the left lobe (indicated by dotted outlines). Scale bar shows $100 \mu \mathrm{m}$. (b-c) UMAP plots of cell cycle-corrected embryonic lung cells isolated by scRNA-seq of E11.5 CD1 mouse lungs, color-coded either by cluster (b) or by replicate (c). (d) UMAP plots from (b-c) color-coded to show the expression of population-specific genes. (e) Top 20 markers for each cluster shown in Fig. 1b based on log fold-change (FC) and color-coded by adjusted p-value.

\section{Figure 2. Computationally identified mesenchymal clusters represent spatially distinct} populations. (a-b) E11.5 lungs immunostained for cluster 0 marker Lef1 (a) or for cluster 1 marker Foxp1 (b) and counterstained with Hoechst. Scale bars show $50 \mu \mathrm{m}$. (c) Violin plots showing the expression of Lefl or Foxpl in each cluster. (d) Schematic depicting the subepithelial and sub-mesothelial compartments of the mesenchyme. (e) Heatmap showing the expression of genes specific to either mesenchymal compartment. Genes (rows) are clustered based on the dendrogram to the right. Cells (columns) are clustered based on the dendrogram above, and each column is color-coded according to original cluster identity from Fig. 1b. (f) UMAP of mesenchymal and smooth muscle cells color-coded according to the sum of their expression of either sub-epithelial or sub-mesothelial mesenchyme marker genes.

\section{Figure 3. Wnt signaling regulates cell identity in the embryonic pulmonary mesenchyme.}

(a) Bubble plot showing the enrichment percentage and adjusted p-value of relevant GO terms identified based on genes upregulated in each mesenchymal compartment. (b) Heatmap showing the expression of Wnt-associated genes upregulated in either mesenchymal cluster. Activators and targets are colored in green, inhibitors are colored in orange. (c) Heatmap showing the 
expression of Wnt ligands, secreted inhibitors, and receptors detected in either mesenchymal cluster and in clusters containing cells from the mesothelium (meso), vascular endothelium (ve), epithelium (ep), and smooth muscle (sm). (d-g) Confocal sections and quantifications of Lef1 and Foxp1 intensity profiles around branch L.L2 in lungs isolated at E11.5 from CD1 embryos and immunostained for Lef1 or Foxp1 after treatment with either DMSO, LiCl (10 mM), or IWR1 $(100 \mu \mathrm{M})$ for $24 \mathrm{hr}(\mathrm{n}=2-6)$. (h-k) Confocal sections and quantifications of Lef1 and Foxp1 intensity profiles around branch L.L2 in lungs isolated at E11.5 and treated with DMSO or cyclopamine $(1 \mu \mathrm{M})$ for $24 \mathrm{hr}(\mathrm{n}=4-5)$. (l-o) Confocal sections and quantifications of Lef1 and Foxp1 intensity profiles around branch L.L2 in lungs isolated at E11.5 and treated with DMSO or a combination of cyclopamine and $\mathrm{LiCl}$ for $24 \mathrm{hr}(\mathrm{n}=2-3)$. Yellow dashed lines indicate border of the epithelium. Schematics show lines and direction along which intensity profiles were measured. Mean and s.e.m. are plotted, and curves were compared using two-way ANOVA. Scale bars show $25 \mu \mathrm{m} . *$ indicates $p<0.05$, ** indicates $p<0.001$, and $* * *$ indicates $p<0.0001$.

Figure 4. Embryonic pulmonary mesenchymal cells exhibit spatially patterned migratory behaviors and cortical tension. (a-c) Confocal sections of Dermo1-Cre/+;Confetti/+ lungs isolated at $E 11.5$, cultured for $48 \mathrm{hrs}$ under time-lapse conditions, and immunostained for Lef1, Foxp1, or $\alpha$ SMA and GFP. (d) Mean speed of tracked cells of each mesenchymal cell type ( $\mathrm{n}=6$ time-lapses, $n=280$ cells total). Error bars show s.d. (e-h) Snapshots from time-lapse analysis showing a mesenchymal cell migrating from the sub-mesothelial to the sub-epithelial mesenchyme (e) and vice versa (g), and percentage of tracked cells from the sub-mesothelial (f) or the sub-epithelial (h) mesenchyme that stay within their original compartment, cross compartments, or elongate, presumably differentiating into smooth muscle. (i) Snapshots from time-lapse analyses of Dermo1-Cre/+;Confetti/+ lungs isolated at E11.5 showing sub-epithelial 
mesenchymal cells differentiating into smooth muscle. (j) Percentage of tracked smooth-muscle cells that were already smooth muscle or that were observed to differentiate from sub-epithelial mesenchymal cells. Yellow arrowhead indicates tracked cell, dashed line indicates the epithelium and dotted line indicates the mesothelium. (k) Schematic of VinTS. Vh is vinculin head, Vt is vinculin tail. (l) Fluorescence intensity of VinTS in E12.5 lung and lifetime of TFP donor along mesenchymal cell edges. (m) Fluorescence lifetime of sub-epithelial and submesothelial mesenchymal cells ( $n=6$ samples, $n=261-282$ cells). Box shows $25^{\text {th }}$ and $75^{\text {th }}$ percentiles, center line shows median. Scale bars show $50 \mu \mathrm{m}$. Groups were compared using two-sided t-test and Bartlett's test for unequal variances. $* * *$ indicates $p<0.0001$ for t-test and ${ }^{\#}$ indicates $p<0.05,{ }^{\# \#}$ indicates $p<0.001$, and ${ }^{\# \#}$ indicates $p<0.0001$ for Bartlett's test.

\section{Figure 5. Diffusion analysis reveals differentiation trajectory of mesenchymal cells into}

airway smooth muscle. (a) Diffusion plots of sub-epithelial mesenchymal and smooth-muscle cells. (b) Scaled expression of known smooth-muscle genes versus cell loadings along DC1. (c) Number of genes detected per cell versus DC1. (d) Heatmap showing 5 gene sets identified using hierarchical agglomerative clustering of spline-fitted gene-expression profiles along DC1. Genes of interest are indicated to the right. (e) Bubble plot showing the enrichment percentage and adjusted p-value of relevant KEGG pathways for the gene sets defined in e. (f) Bubble plot showing the $\mathrm{p}$-values of motifs for transcription factor-binding sites in the promoters of and DNase-hypersensitive regions near genes of sets 2-5. Pearson correlation coefficients and significance are indicated on plots and lines represent smoothed data with standard error shaded in gray. $* * *$ indicates $p<0.0001$

Figure 6. Novel regulators and features of smooth-muscle differentiation. (a) Sections of E12.5 Dermo1-Cre/+;Yap-fl/fl;mTmG/+ lungs and littermate controls immunostained for GFP 
and either Yap1, Lef1, Foxp1, or aSMA. (b) Scaled expression of genes involved in cytoskeleton, cell adhesion, and extracellular matrix versus cell loadings along DC1 compared to the expression profiles of smooth-muscle genes Acta2 and Myocd (dotted lines). Pearson correlation coefficients and significance are indicated and lines represent smoothed data with standard error shaded in gray. (c) Simplified pathway diagram depicting the steps of proliferative metabolism and showing relevant enzymes at each step. Enzymes that are significantly downregulated along DC1 are indicated in bold red font, with significance of spline fit indicated by asterisks. * indicates $p<0.05, * *$ indicates $p<0.001$, and $* * *$ indicates $p<0.0001$.

\section{Figure 7. Wnt signaling regulates the accumulation of F-actin and nascent smooth-muscle} cells in the sub-epithelial mesenchyme to influence epithelial morphology. (a) Confocal sections of the left lobe of lungs isolated from E11.5 CD1 embryos and immunostained for Ecadherin (Ecad) and $\alpha$ SMA after treatment with DMSO, LiCl, or IWR1 for 24 hrs. (b-c) Quantification of smooth-muscle coverage around the left bronchus proximal or distal to L.L2 (see dashed white lines in a, n=5-10). (d) Number of new branches formed in the left lobe after $24 \mathrm{hrs}$ of culture for each treatment condition. (e) Schematic showing where branch width and length were measured. (f-g) Width and length of L.L2 after 24 hrs of culture for each treatment condition. Error bars show SD. Treatment conditions were compared using one-way ANOVA. (h) Confocal sections of the left lobe of lungs isolated from E11.5 SMA-RFP embryos and immunostained for RFP and labelled with phalloidin after treatment with DMSO or LiCl for 24 hrs. (i) Quantification of F-actin intensity profiles emanating from the epithelium (as depicted in the schematic) for each treatment. Curves were compared using two-way ANOVA. * indicates $p<0.05$ and $* * *$ indicates $p<0.0001$. 


\section{Supplementary Information}

\section{Supplementary Figure Legends}

\section{Supplementary Figure 1 - related to Figure 1. scRNA-seq experimental design, summary} statistics, and initial clustering. (a) Images of all E11.5 lungs used for the two replicates of the scRNA-seq experiment. Each lung was micro-dissected to isolate the left lobe (indicated by dotted outlines). Scale bars show $100 \mu \mathrm{m}$. (b) Table of general statistics for each sample. UMI (unique molecular identifiers) reflects the number of transcripts captured per cell. (c-d) UMI counts as a function of number of barcodes for replicates 1 (c) and 2 (d). Portion of curve above threshold includes all cells (green) and portion below is considered background (grey). (e) Table of sequencing statistics for each replicate. Q30 statistics give the percentage of bases in the indicated sequence type with a quality score greater than 30. (f-g) Sequencing depth as a function of mean reads per cell for each replicate. (h-i) Median genes per cell as a function of mean reads per cell. (j) Table of mapping statistics for each replicate. Mapping was performed using STAR. (k-l) UMAP plot of all cells isolated by scRNA-seq of E11.5 CD1 mouse lungs, color-coded by cluster (k) or by replicate (1). (m-n) UMAP plot after all blood and immune cells have been removed, color-coded by cluster (m) or by replicate (n).

\section{Supplementary Figure 2 - related to Figure 1. Pulmonary mesenchymal cells are}

heterogeneous but ultimately closely related. (a-c) UMAP plots of isolated mesenchymal cells color-coded to show the expression of key markers from Fig. 1: Hoxb6, Ptn, and Acta2. (d-g) UMAP from (a) color-coded to show the expression of putative smooth muscle progenitor markers Axin2, Fgf10, Gli1, and Wt1. (h) UMAP of mesenchymal and smooth muscle cells corrected for cell-cycle stage and color-coded based on cluster identified by Seurat. (i-m) Top 20 marker genes for each cluster based on average log fold-change (FC) and color-coded by 
adjusted p-value. (n-q) Violin plots showing the expression of putative smooth muscle

progenitor markers Axin2, Fgf10, Gli1, and Wt1 in each of the mesenchymal clusters represented in $(\mathrm{h})$.

\section{Supplementary Figure 3 - related to Figure 2. Lef1 and Foxp1 expression patterns}

throughout the lung and across developmental stages. (a-b) Zoomed-in view of a confocal slice of the left primary bronchus of lungs isolated at E12.5 and immunostained for $\alpha \mathrm{SMA}$ and either Lef1 or Foxp1. Epithelium indicated by ep. (c-f) Confocal slices of the left lobe of lungs isolated at E12.5 or E14.5 and immunostained for either Lef1 or Foxp1 and counterstained with Hoechst. Scale bars show $50 \mu \mathrm{m}$.

\section{Supplementary Figure 4 - related to Figure 3. Manipulating Wnt signaling ex vivo alters}

levels of Axin2 and $\beta$-catenin in the pulmonary mesenchyme. (a-b) Sections of lungs isolated at $E 11.5$ from $\mathrm{CD} 1$ embryos and immunostained for Axin2 or $\beta$-catenin and counterstained with Hoechst after treatment with either DMSO, $\mathrm{LiCl}(10 \mathrm{mM})$, or IWR1 $(100 \mu \mathrm{M})$ for $24 \mathrm{hrs}$. (c) Quantification of mean cytoplasmic Axin2 immunofluorenscence intensity in the mesenchyme for each treatment ( $\mathrm{n}=4-11$ sections, $n=2-3$ lungs). (d) Quantification of the ratio of nuclear to cytoplasmic $\beta$-catenin immunofluorenscence intensity in the mesenchyme for each treatment ( $\mathrm{n}=3-7$ sections, $\mathrm{n}=2-3$ lungs). Scale bars show $50 \mu \mathrm{m}$. Treatment conditions were compared using one-way ANOVA. * indicates $p<0.05$ and $* *$ indicates $p<0.001$

\section{Supplementary Figure 5 - related to Figure 4. Confetti fluorescent reporters driven by}

\section{Dermo1-Cre can be used to track single-cell movements in the embryonic pulmonary}

mesenchyme. (a) Confocal slice of the left lobe of lungs isolated at E11.5 from a Dermo1-

Cre/+;mTmG/+ embryo and immunostained for E-cadherin and GFP. GFP expression

throughout the mesenchyme indicates that Cre is active throughout this tissue. (b) Snapshots 
from time-lapse analysis of Dermo1-Cre/+;Confetti/+ lung isolated at E11.5 showing a bifurcating epithelial bud surrounded by sparsely fluorescently-labelled mesenchymal cells. (c-d) Snapshots from a time-lapse of Dermo1-Cre/+;Confetti/+ lung isolated at E11.5 (c) and plot of cell tracks (d). Tracks colored in gray are uncorrected and tracks colored in blue are corrected for local tissue movement. Cell-type assignments are indicated by the shaded regions. (e-f) Distributions of instantaneous speeds from each cell track (e) and of total cell displacements before and after correcting for local tissue movement. (g) Log-log plot of mean MSD curves of each mesenchymal cell type. Error bars show s.d. (h) Persistence of cell tracks measured as slope of MSD curves from (g). (i) Fluorescence lifetime of each lung tissue. Data points represent average per sample. Box shows $25^{\text {th }}$ and $75^{\text {th }}$ percentiles, center line shows median. (j) VinTS lung stained for PECAM. Cells with high VinTS expression dispersed throughout the mesenchyme are vascular endothelial cells. Scale bars show $50 \mu \mathrm{m}$. Groups were compared using two-sided t-test and Bartlett's test for unequal variances. *** indicates $p<0.0001$ and *

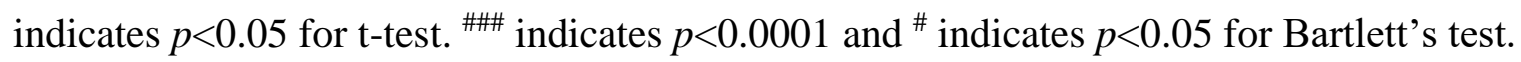

\section{Supplementary Figure 6 - related to Figure 5. Sub-mesothelial cells may preferentially give}

rise to vascular smooth muscle cells. (a) Table showing the expected expression of each of marker in airway/visceral smooth muscle versus vascular smooth muscle ${ }^{3}$. (b-h) Violin plots showing the scaled expression of Actg2, Foxf1, Gata5, Heyl, Mylk, Nog, and Speg in each of the mesenchymal and smooth-muscle clusters from Fig. 1b. Gene expression per cell cluster compared by Wilcoxon Rank Sum Test using the FindMarkers function. (i-p) UMAP of smooth muscle cells computationally isolated from the dataset in Fig. 1b color-coded based on scaled expression of marker genes for airway and vascular smooth muscle. (q-s) Sections of E14.5 lungs immunostained for E-cadherin, $\alpha$ SMA, and either PECAM, Lef1, or Foxp1 and 
counterstained with Hoechst. Airway smooth muscle (ASM) and vascular smooth muscle (VSM) are indicated. Scale bars show $50 \mu \mathrm{m}$. * indicates $p<0.05$, ** indicates $p<0.001$, and *** indicates $p<0.0001$.

\section{Supplementary Figure 7 - related to Figure 5. Transcription factors identified in motif} discovery analysis are expressed in the embryonic lung. Motif logos and tissue-specific expression of corresponding transcription factors for results presented in Fig. 5f. For expression of Lef1 and Foxp1, see Fig. 2c.

\section{Supplementary Figure 8 - related to Figure 6. The majority of smooth muscle marker}

genes are Myocd-independent. (a) Volcano plot showing the adjusted p-values and log fold changes of gene-set members (Fig. 5d) that were detected in bulk RNA-seq of E13.5 Myocd-KO lungs compared to controls. Insets to show each gene set separately with the percentage of genes in each quadrant of the volcano plot indicated. (b) Adjusted p-values plotted against the mean of regularized log-transformed counts across all samples showing that genes that were not significantly different were detected at similar levels as genes that were significantly different. Points are color-coded according to gene set and insets to the right show each gene set separately. (c) Volcano plot showing the adjusted p-values and log fold changes of smoothmuscle cluster markers (Fig. 1b, e) that were detected in bulk RNA-seq of E13.5 Myocd-KO lungs compared to controls. The percentages of genes in each quadrant of the volcano plot are indicated. (d) Adjusted p-values plotted against the mean of regularized log-transformed counts across all samples. 


\section{Supplementary Tables}

Supplementary Table 1. Markers for clusters of embryonic lung cells identified in Fig. 1.

Supplementary Table 2. Genes that are differentially expressed in either sub-epithelial or submesothelial mesenchymal cells relative to each other.

Supplementary Table 3. Curated list of genes from the MGI database that are expressed in the embryonic pulmonary mesenchyme.

Supplementary Table 4. GO enrichment analysis results for genes that are differentially expressed in either sub-epithelial or sub-mesothelial mesenchymal cells relative to each other.

Supplementary Table 5. Gene sets identified in the diffusion analysis of smooth muscle differentiation in Fig. 5.

Supplementary Table 6. KEGG pathway enrichment analysis results for gene sets identified in the diffusion analysis of smooth muscle differentiation in Fig. 5.

\section{Supplementary Videos}

Supplementary Video 1. Time-lapse imaging of lungs isolated from a Dermo1-Cre/+;Confetti/+ embryo at $E 11.5$. 
Figure 1

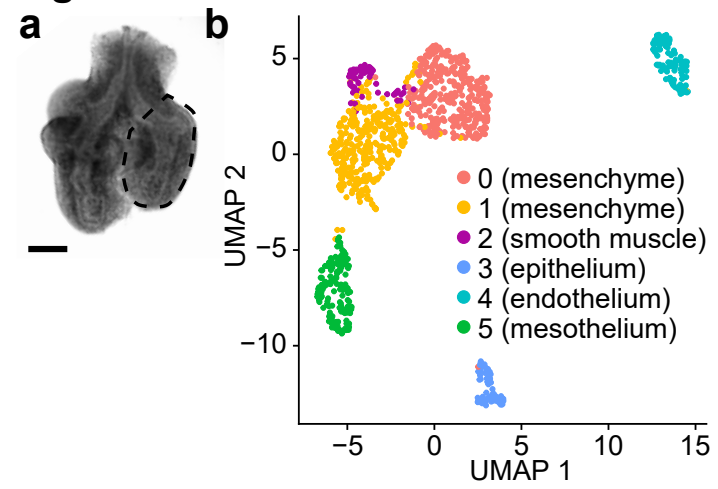

e cluster 0: mesenchyme average log FC

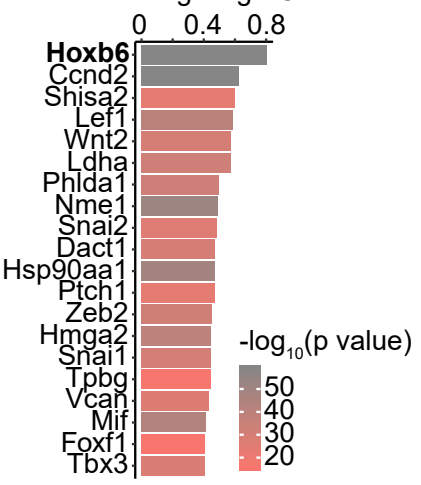

cluster 4: vascular endothelium average log FC

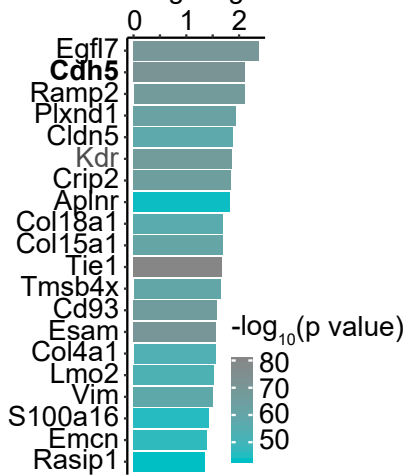

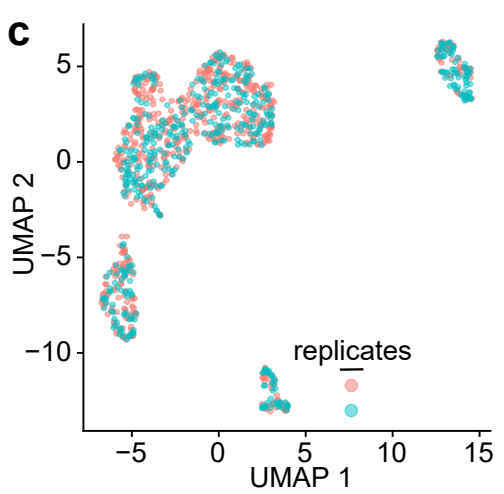

cluster 2: smooth muscle
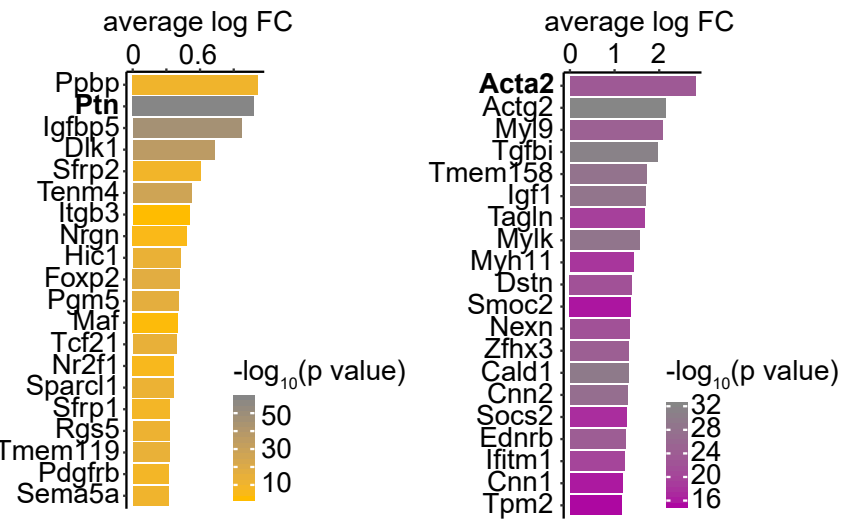

cluster 3: epithelium average log FC 00.511 .5

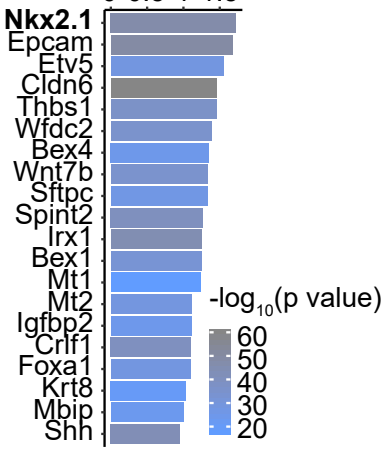

d Hoxb6

scaled expression
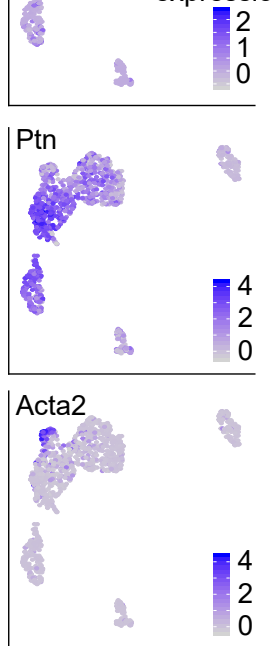

Nkx2.1
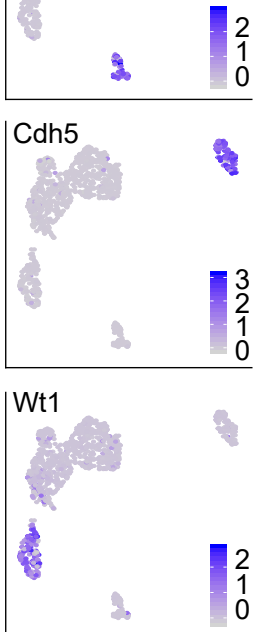
Figure 2
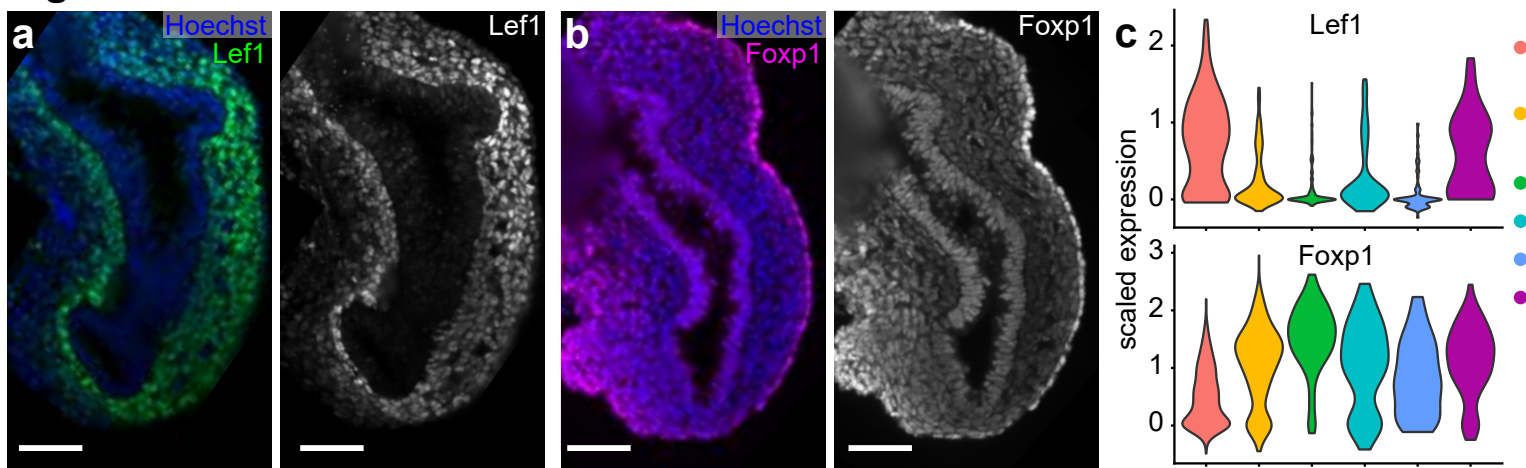

- 0 (sub-epithelial mesenchyme)

1 (sub-mesothelial mesenchyme)

2 (mesothelium)

- 3 (endothelium)

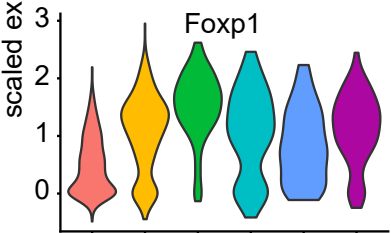

- 4 (epithelium)

- 5 (smooth muscle)

d

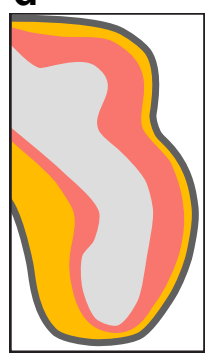

epithelium

sub-epithelial mesenchyme

sub-mesothelial mesenchyme

mesothelium

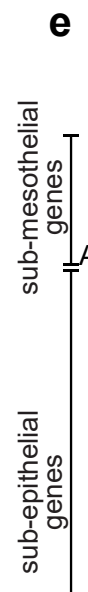

e

scaled expression $\begin{array}{ccccccc}-6 & -4 & -2 & 0 & 2 & 4 & 6\end{array}$
$18 \%$ of cells in cluster 0 $82 \%$ of cells in cluster 1

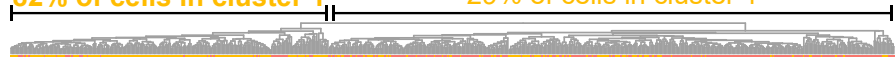
Pitx2 Osr1 Sfrp2 Aldh1a2 Wr2f1 Lef1 Col4a1 Axin2 Tgfbil 1 Tgfbi Foxf2 Tcf7 Emp4 Snai1 Sdc2 Pth1r Hmga2

Tbx3 Fendrr Ptch1

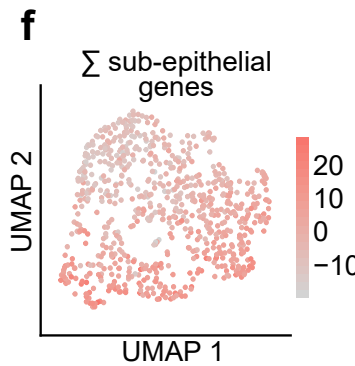

$\sum$ sub-mesothelial genes

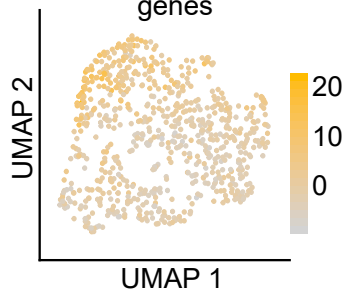


Figure 3

a

GO term

morphogenesis of a branching epithelium regulation of Wnt signaling pathway Wnt signaling pathway cell-cell signaling by Wnt

\% genes $p$ value
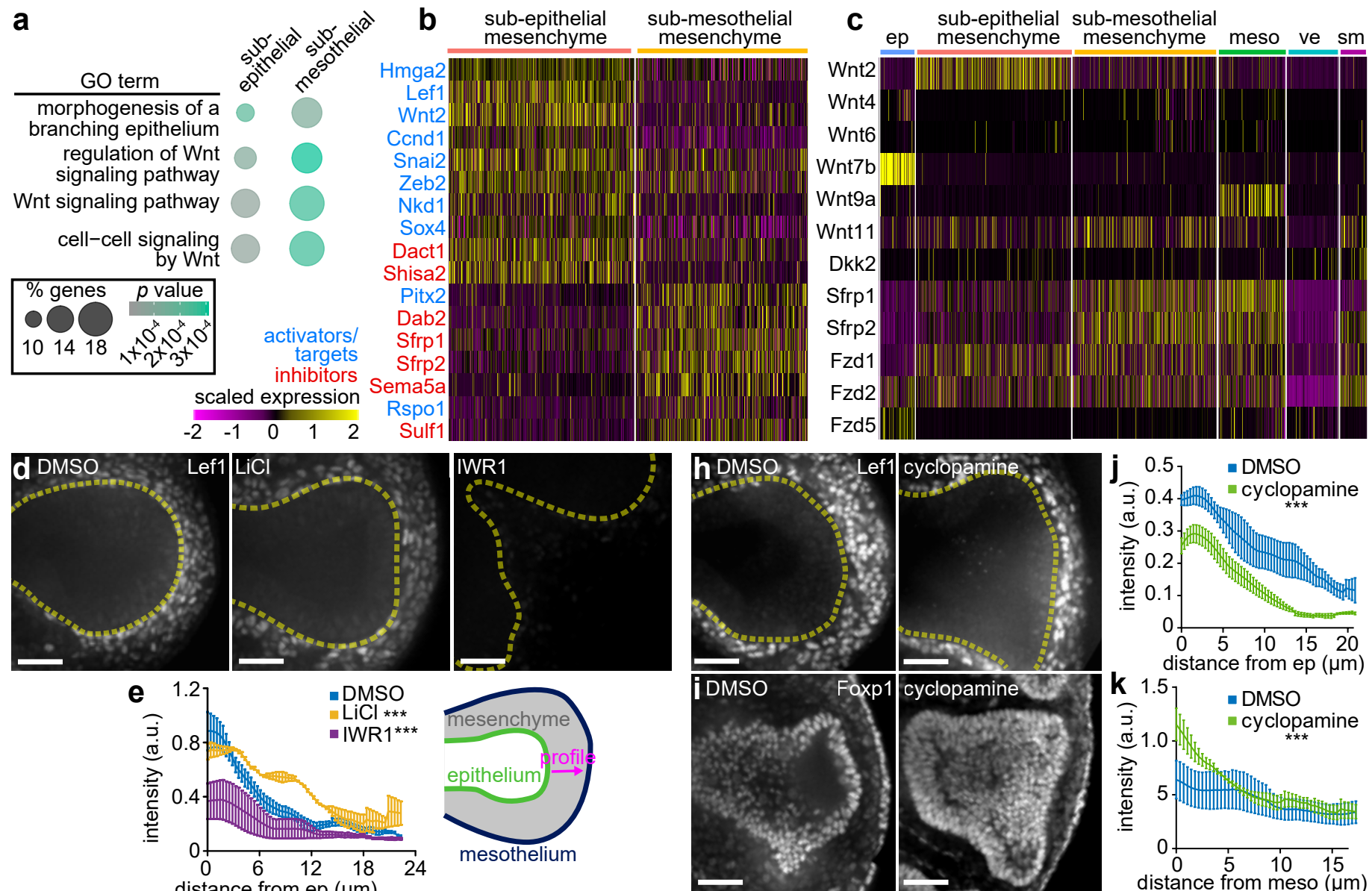
distance from ep ( $\mu \mathrm{m})$
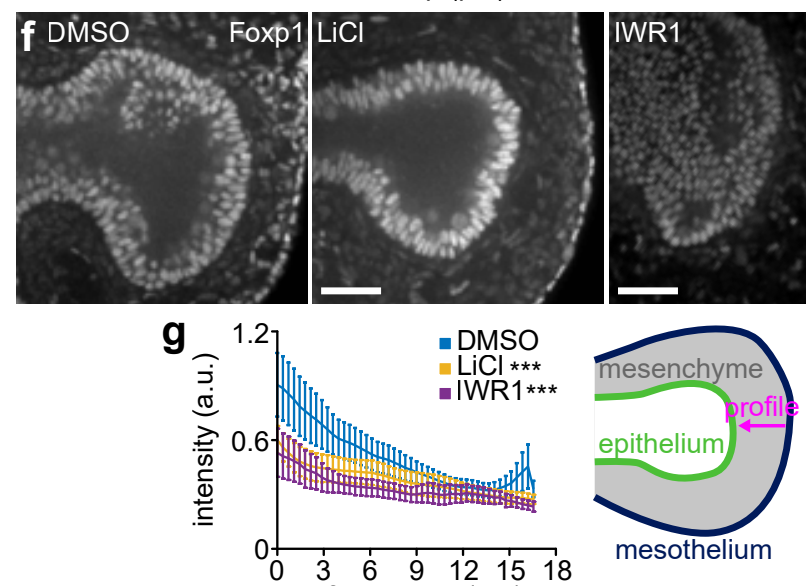
distance from meso $(\mu \mathrm{m})$
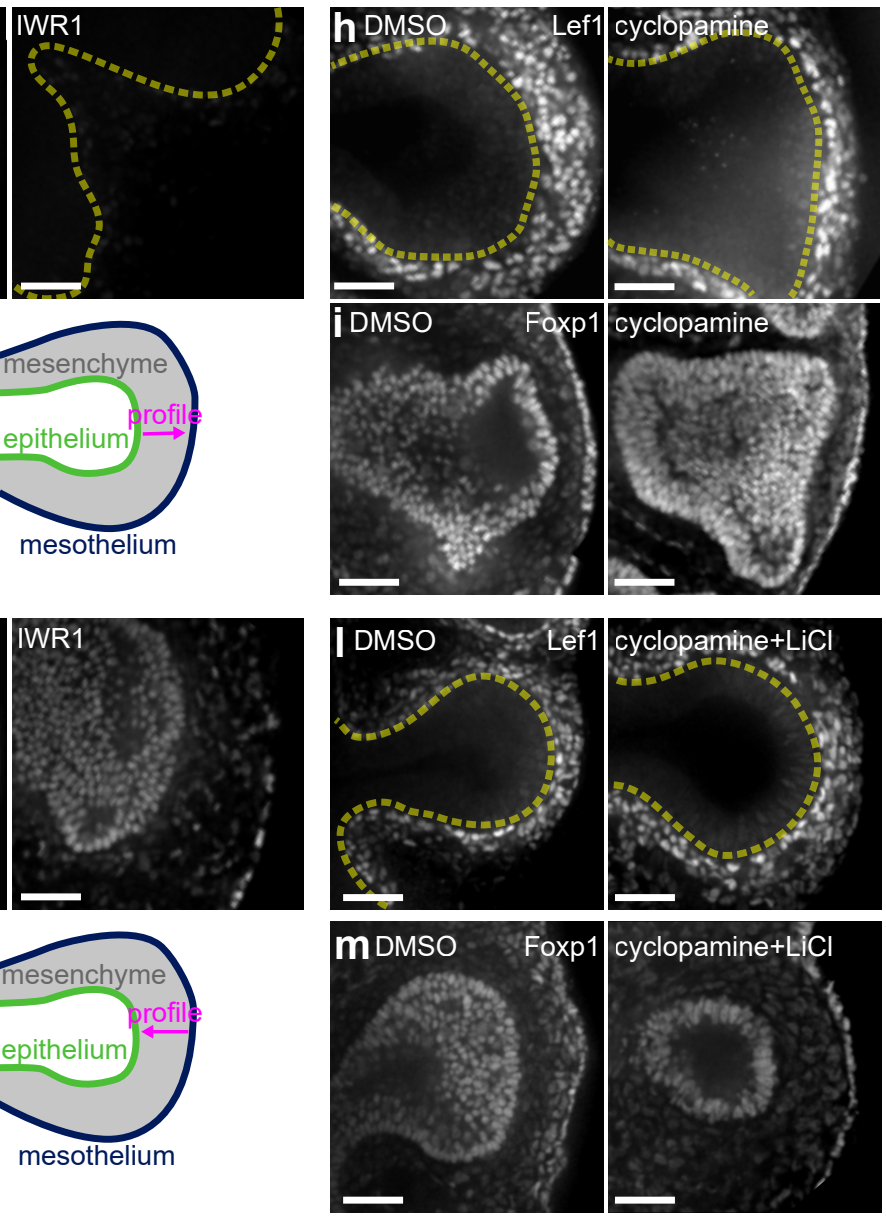
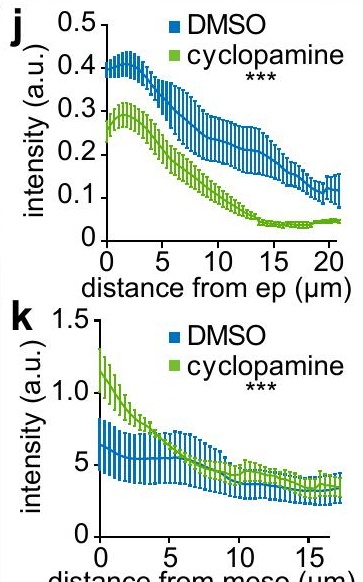

distance from meso $(\mu \mathrm{m})$
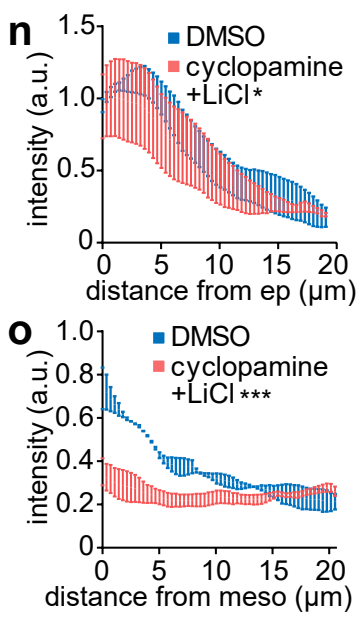
Figure 4

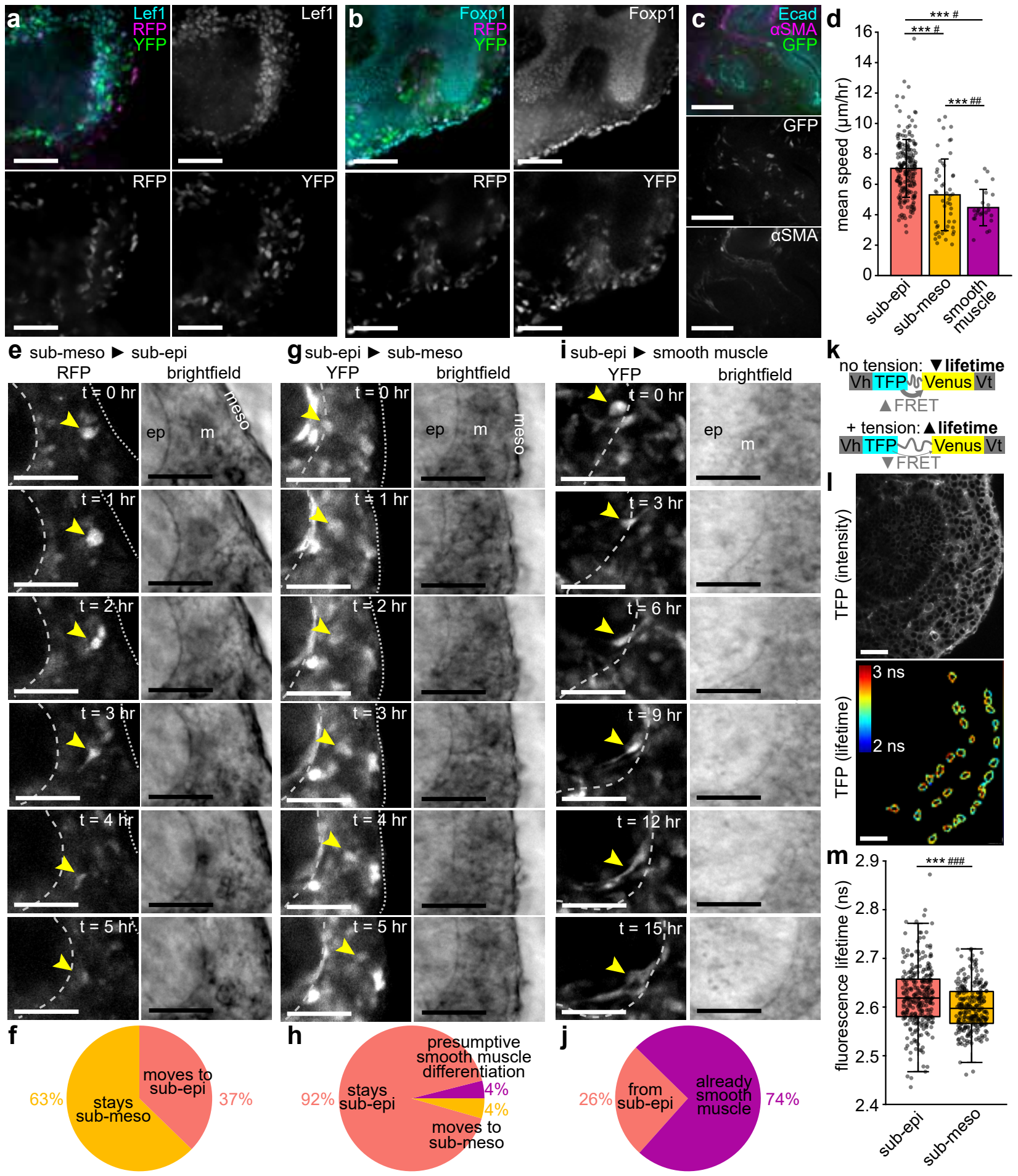




\section{Figure 5}

\section{a}

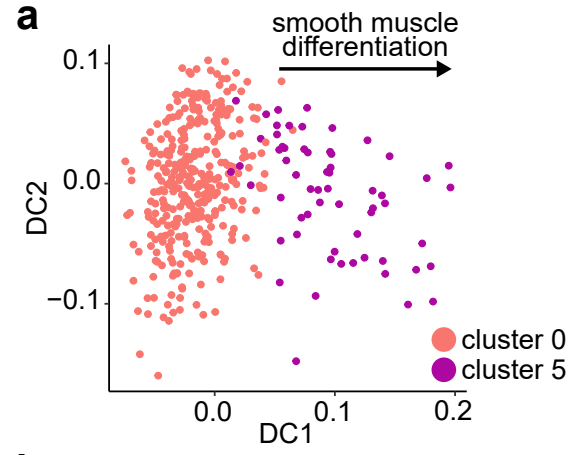

b

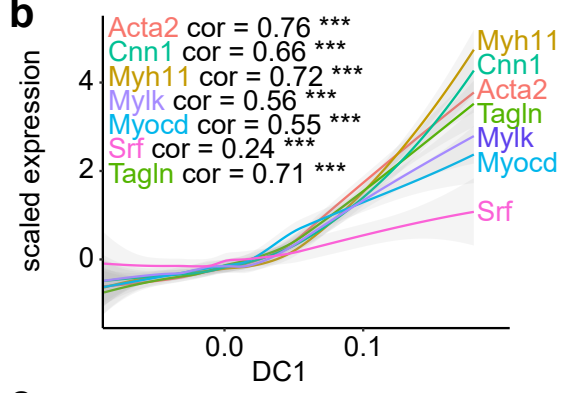

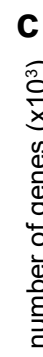

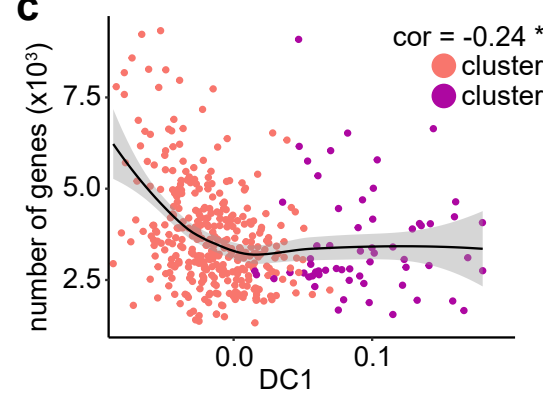

\section{d smooth muscle fitted gene expression}

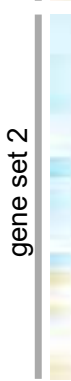

Ctgf

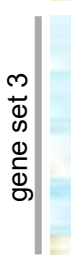

$\checkmark$

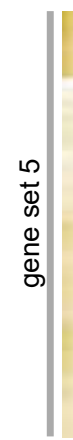

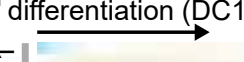

Tagln

Hhip

Acta2
Mylk

Cnn1

Myh11

$\triangle \mathrm{Smtn}$

- Tagln2

$\triangle$ Bmp4

e

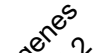

$3 \quad 5 \quad \%$ genes $-\log _{10}(p$ value $)$

\begin{tabular}{|l|} 
Wnt signaling pathway \\
TGF-beta signaling pathway \\
MAPK signaling pathway \\
Hippo signaling pathway
\end{tabular}

$<\mathrm{Cnn} 2$

Myocd

Myl6

Trffbi

$\rightarrow$ Axin2

$<\mathrm{Tbx} 3$

$-\mathrm{Tbx} 2$

\begin{tabular}{|l|}
\hline Carbon metabolism \\
One carbon pool by folate \\
Glycolysis / Gluconeogenesis \\
Biosynthesis of amino acids \\
Cysteine and methionine \\
metabolism \\
Pyrimidine metabolism \\
$\begin{array}{l}\text { Fructose and mannose } \\
\text { metabolism }\end{array}$ \\
\hline
\end{tabular}

f

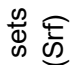



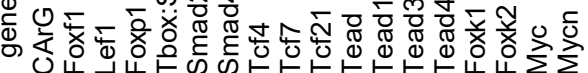
2

$\checkmark$ Wnt2

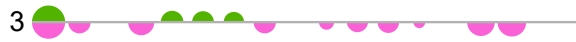

4

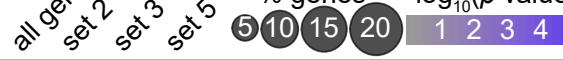

5

promoters

DNase hypersensitive regions 
Figure 6

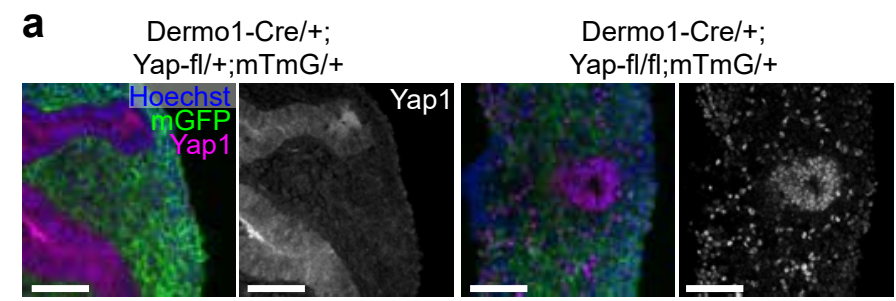

sub-epithelial mesenchyme

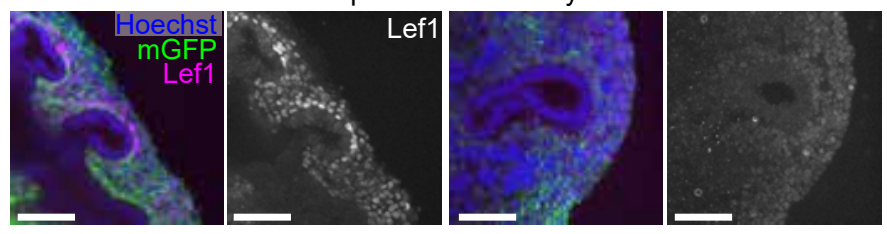

sub-mesothelial mesenchyme

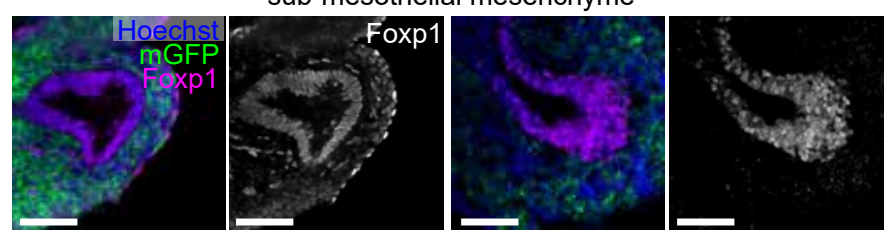

airway smooth muscle

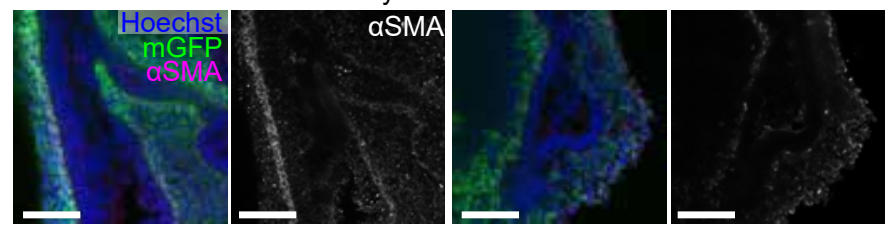

b actin cytoskeleton

cell adhesion
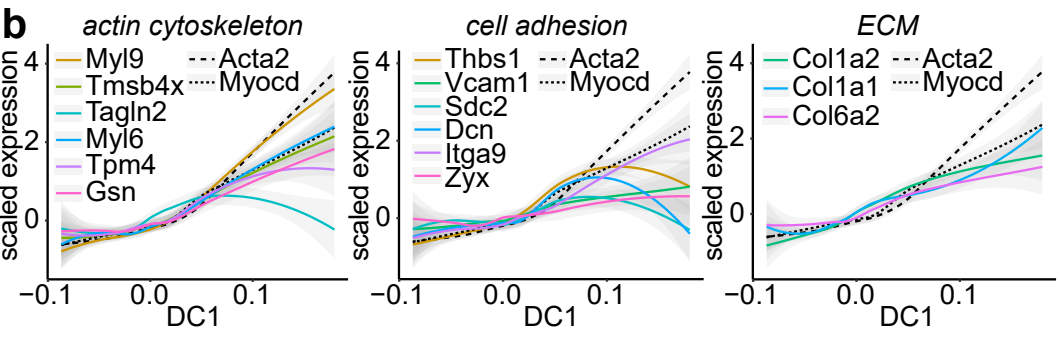

C Glycolysis

Fructose metabolism

$\underset{* *}{A k r 1 b 3}, \ldots$

$\mathrm{Hk} 1, \mathrm{Hk} 2, \ldots$

glucose-6-P

Gpi1

fructose-2,6-2P $\longrightarrow$ fructose-6-P

$$
\begin{aligned}
& \therefore-P f k f b 3 \\
& \text { fructose-1,6-2P }
\end{aligned}
$$

Aldoa

glyceraldèhyde-3-P

Gapdh

1,3-biphosphoglycerate

Pgam 1/2 Serine synthesis pathway

Eno1, ...

phosphoenolpyruvate

Pkm

pyruvate

Ldha, ...

lactate TCA cycle $\rightarrow$ Amino acid

$\mathrm{Fh1}, \mathrm{Mdh} 2 \ldots$
Nucleotide synthesis pathway

ribose-6-P $\longrightarrow$ PRPP

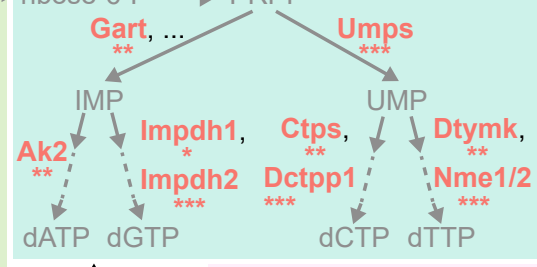

$$
\text { biomass } \rightarrow \text { Folate synthesis pathway }
$$

production $\rightarrow \quad$ Mthfd2, $\rightarrow$

Serine synthesis pathway
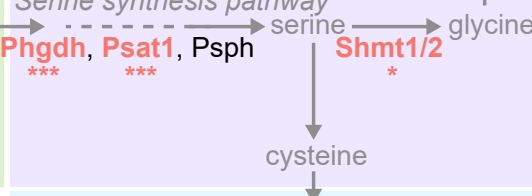

cysteine

1

synthesis

Amd1, Odc1, $\underset{* * * *}{\operatorname{Srm}}, \ldots$ 


\section{Supplementary Figure 1}

a

\begin{tabular}{|l|c|c|}
\hline & \\
\hline b & \\
\hline \multicolumn{1}{|c|}{ General } & \\
\hline
\end{tabular}

C

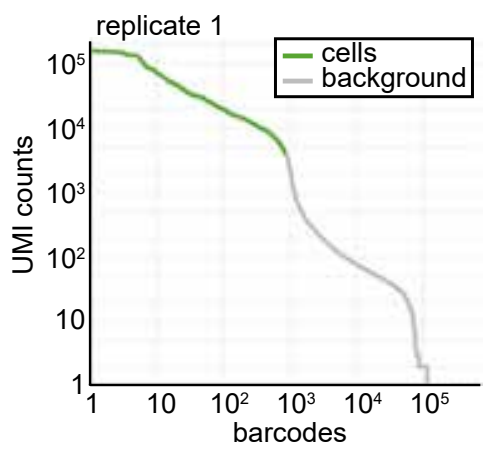

d

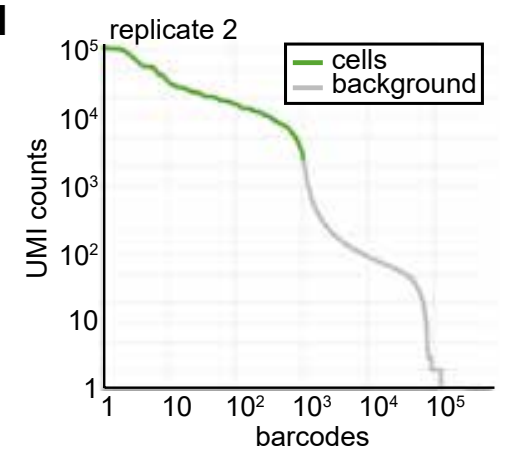

f

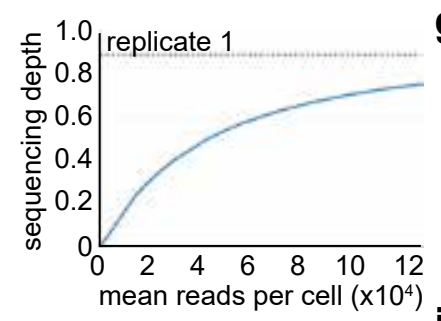

h

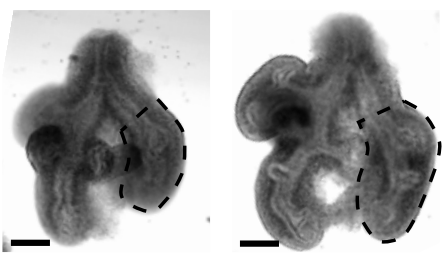

e Sequencing

Number of reads

Valid barcodes

Sequencing saturation

Q30 bases in barcode

Q30 bases in RNA read

Q30 bases in UMI

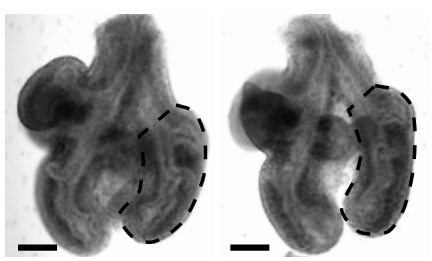

$9{ }^{1.0}$ replicate 2

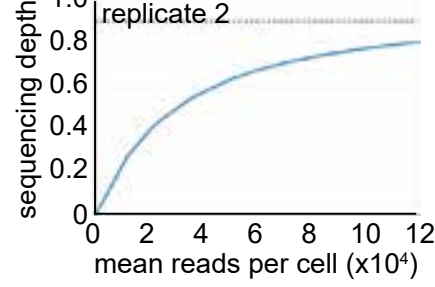

mean reads per cell $\left(x 10^{4}\right)$

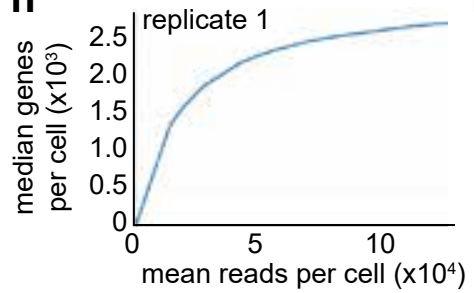

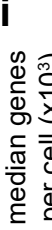

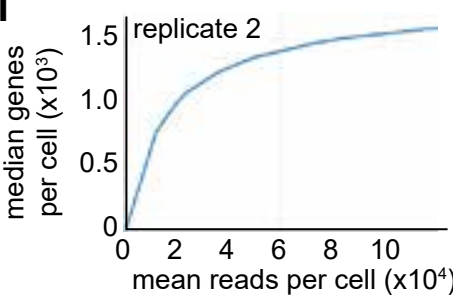

\begin{tabular}{|l|c|c|}
\hline \multicolumn{1}{|c|}{ Mapping } & $\mathbf{1}$ & $\mathbf{2}$ \\
\hline Reads mapped confidently to genome & $73.30 \%$ & $72.10 \%$ \\
\hline Reads mapped confidently to intergenic regions & $5.00 \%$ & $4.40 \%$ \\
\hline Reads mapped confidently to intronic regions & $9.40 \%$ & $7.10 \%$ \\
\hline Reads mapped confidently to exonic regions & $58.90 \%$ & $60.70 \%$ \\
\hline Reads mapped confidently to transcriptome & $57.20 \%$ & $59.30 \%$ \\
\hline Reads mapped antisense to gene & $0.80 \%$ & $0.60 \%$ \\
\hline
\end{tabular}

k
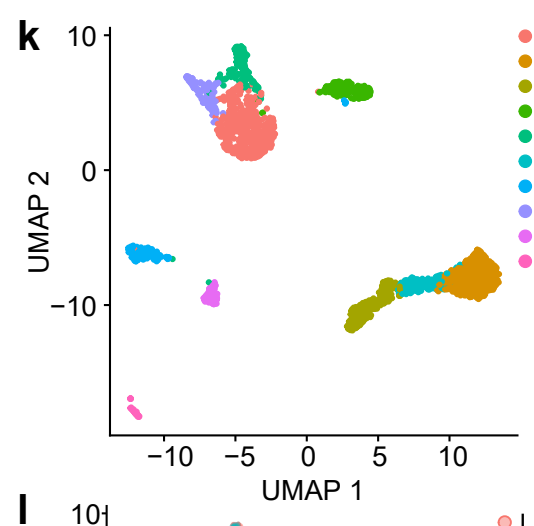

O

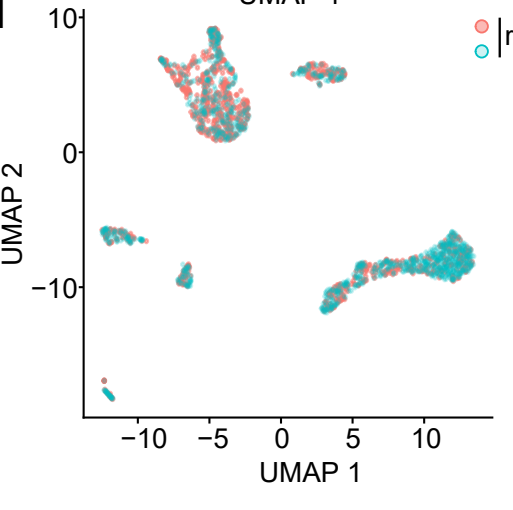

0 (mesenchyme)

1 (blood)

2 (blood)

3 (mesothelium)

4 (mesenchyme)

5 (blood)

6 (endothelium)

7 (smooth muscle)

8 (epithelium)

9 (immune cells)

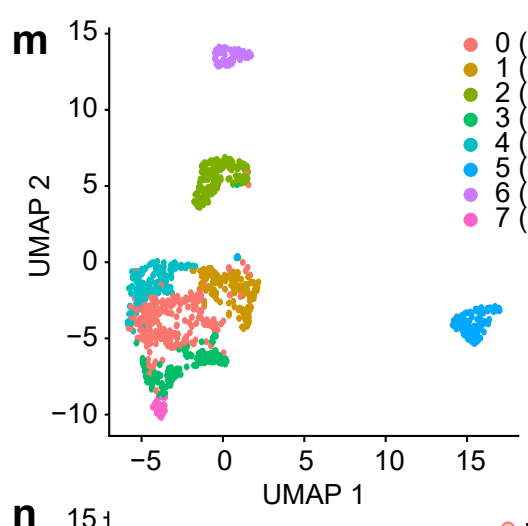

n 15

|replicates

myme

2 (mesothelium)

4 (mesenchyme

5 (endothelium)

(mesenchyme)

7 (smooth muscle)

replicates

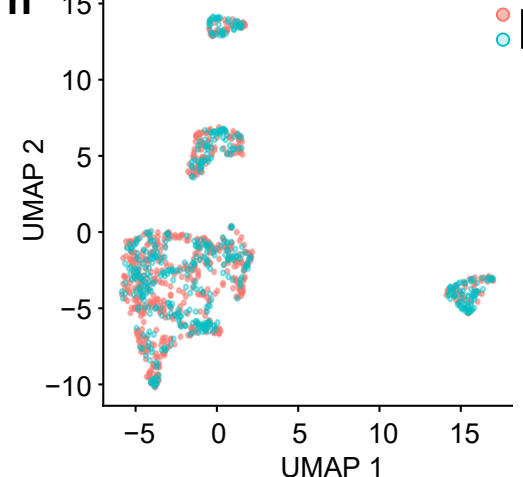



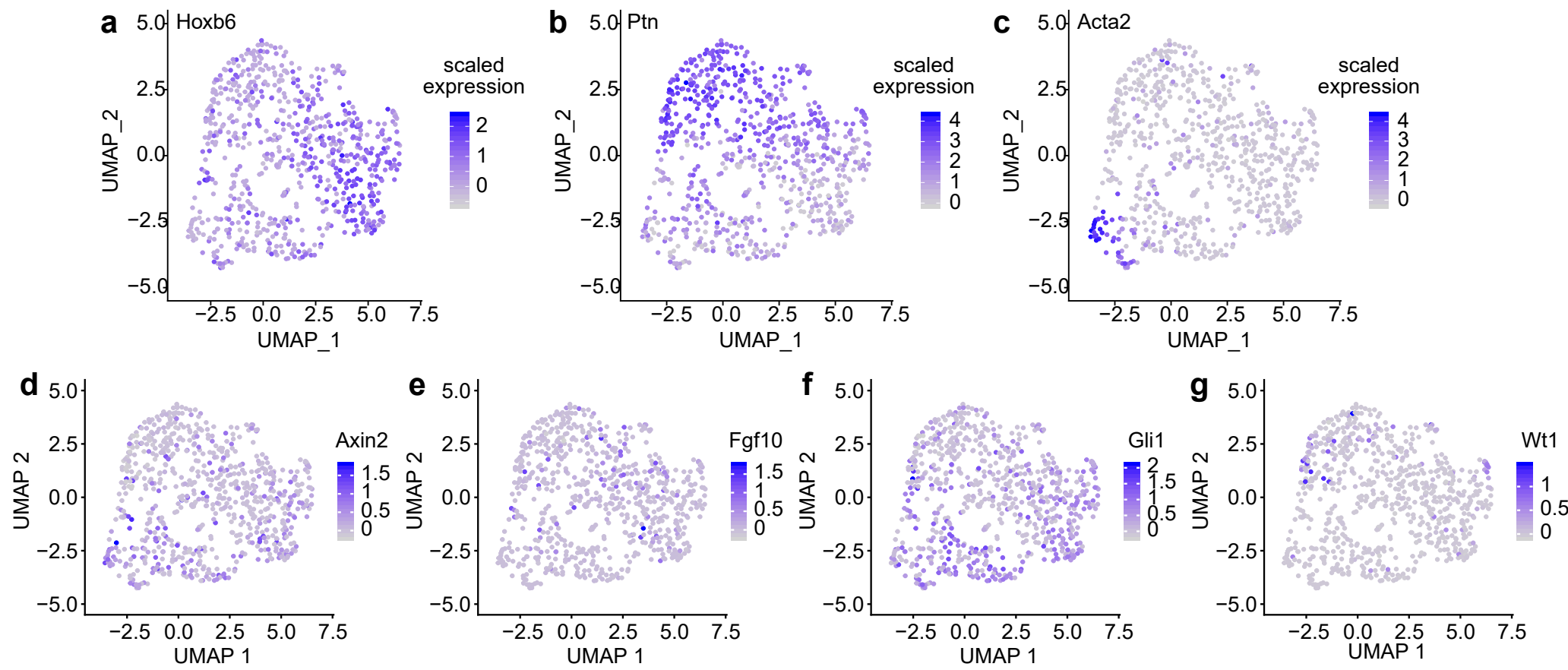

h

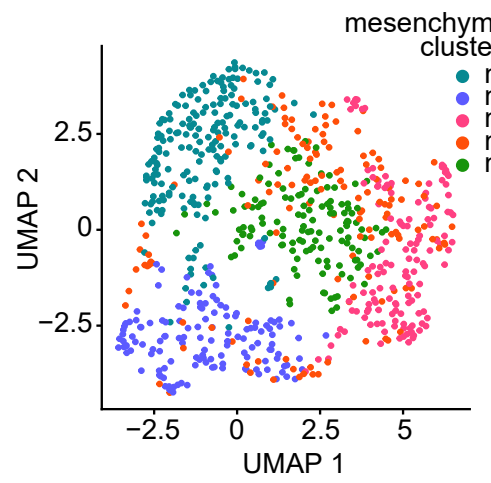

i cluster $\mathrm{m0}$ average log $\mathrm{FC}$
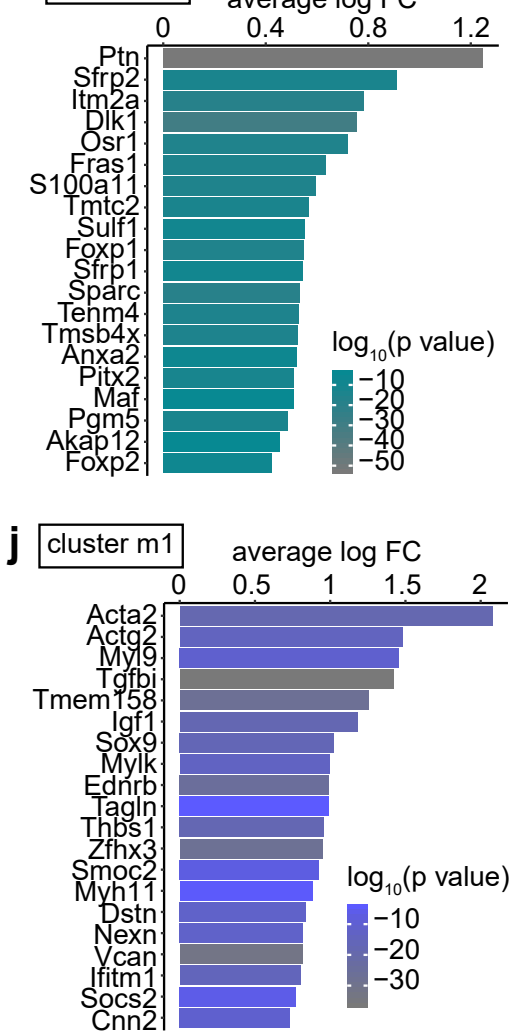

k cluster $\mathrm{m} 2$ average log $\mathrm{FC}$

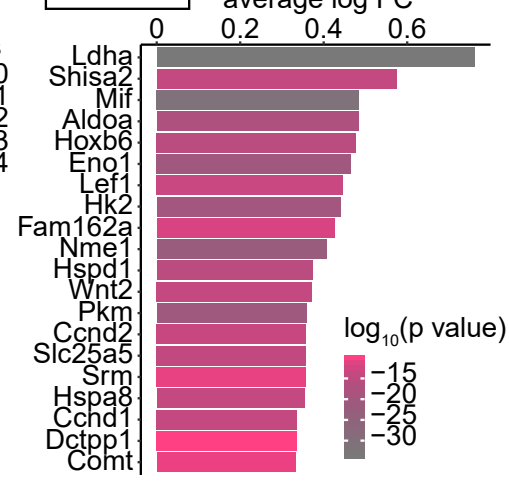

I cluster m3 average log FC

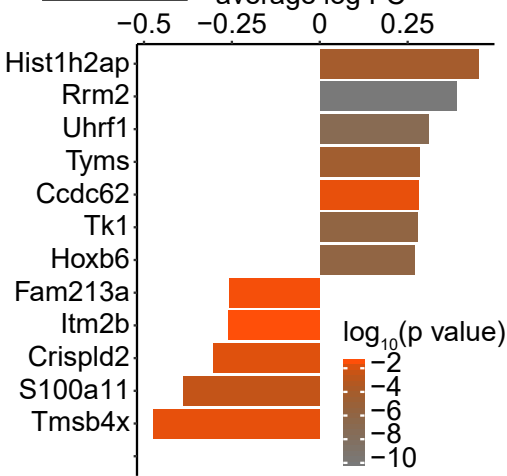

m cluster $\mathrm{m} 4$ average log $\mathrm{FC}$

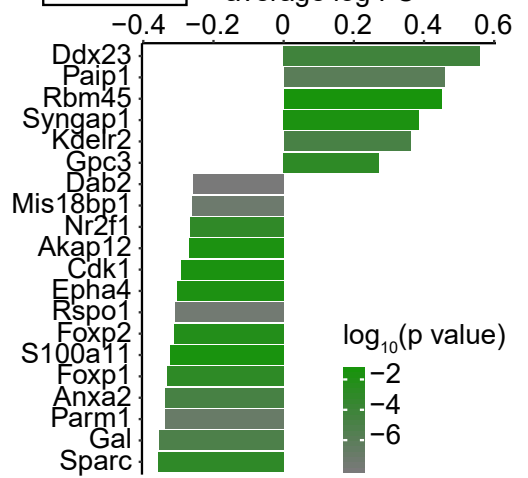

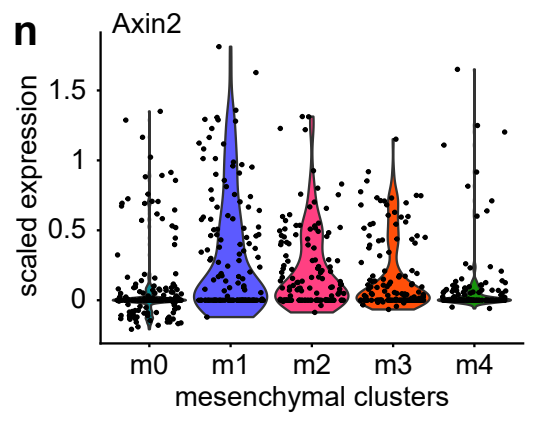
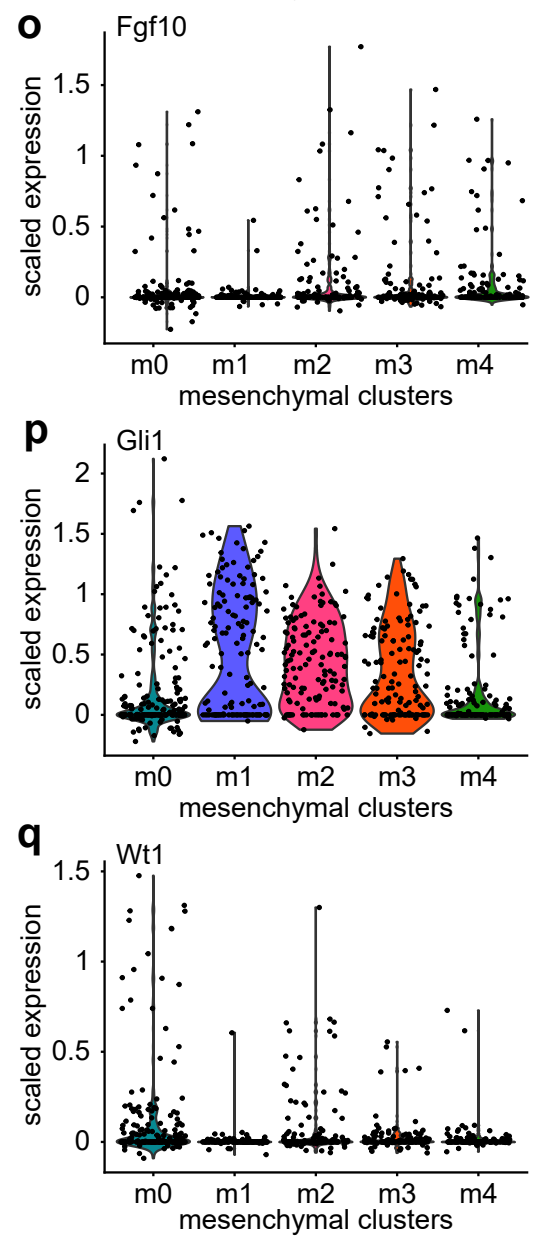


\section{Supplementary Figure 3}
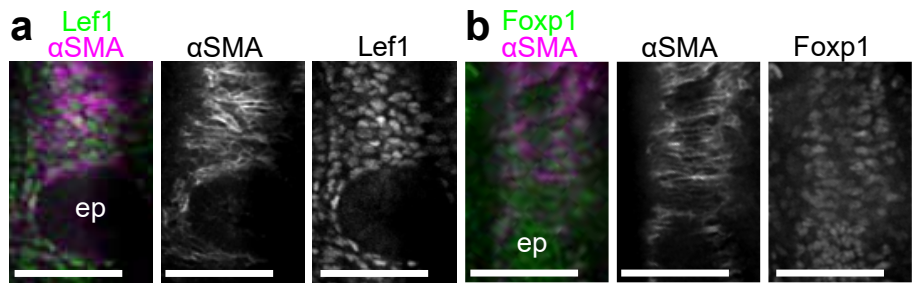

C Hoechst Lef1

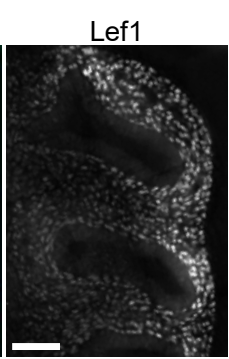

d Hoechst Foxp1

Foxp1

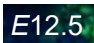
E12.5

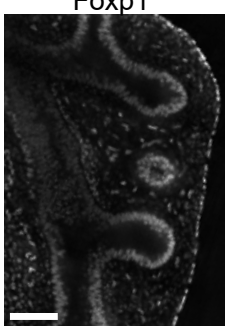

\section{e Hoechst Lef1}

E14.5
Lef1

f Hoechst Foxp1 E14.5
Foxp1

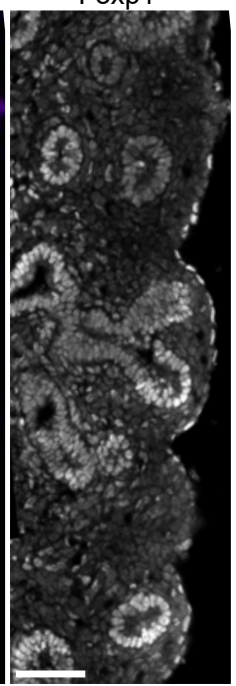


Supplementary Figure 4

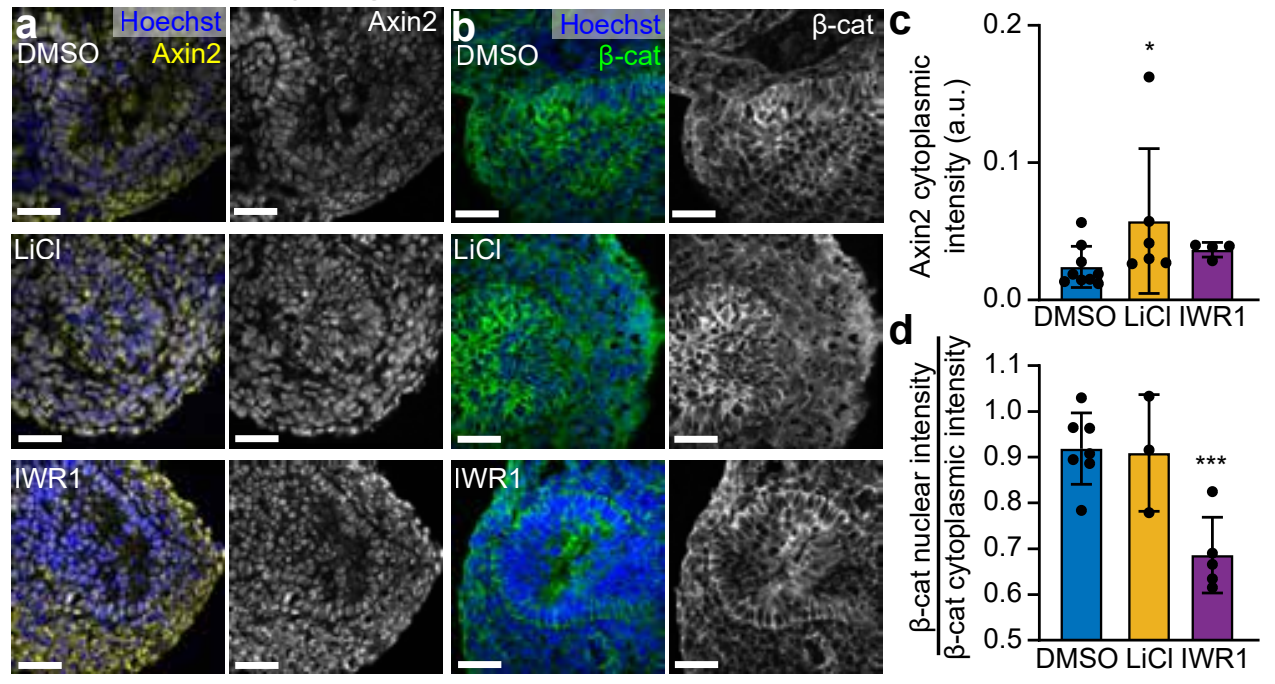


Supplementary Figure 6

\begin{tabular}{l|c|c} 
a & $\begin{array}{c}\text { airway/ } \\
\text { visceral }\end{array}$ & vascular \\
\hline Actg2 & more & less \\
Foxf1 & yes & no \\
Gata5 & yes & no \\
Heyl & no & yes \\
Mylk & more & less \\
Nog & yes & no \\
Speg & less & more
\end{tabular}
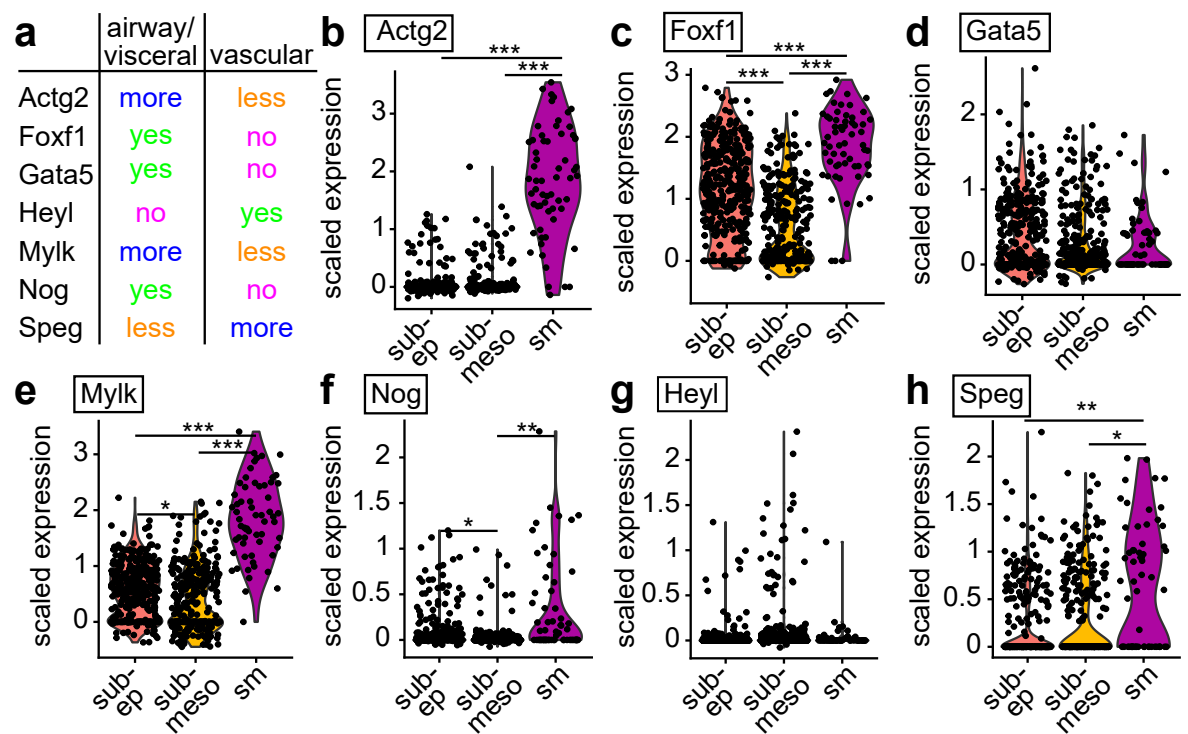

9 Heyl
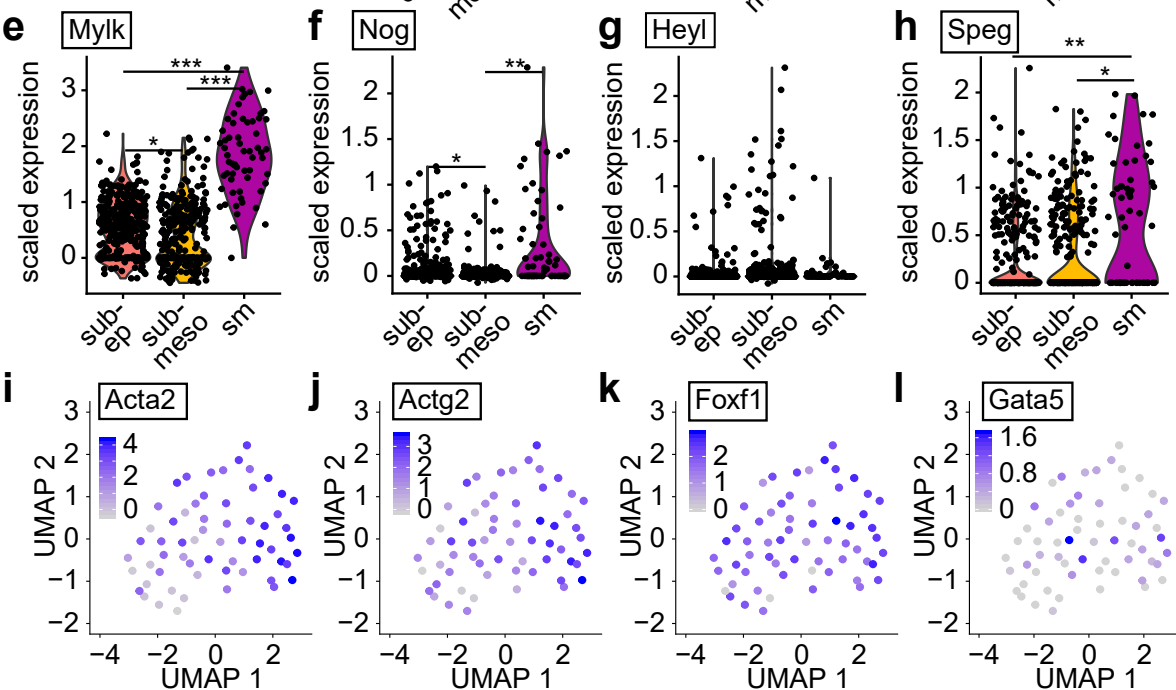

j 3 Actg2

k 3 Foxf1

$\mathrm{N}^{2} \mathrm{D}_{2}^{3} \quad \cdots$

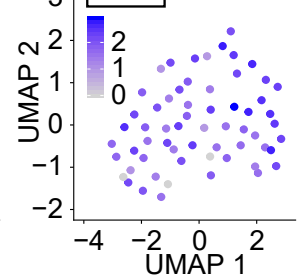

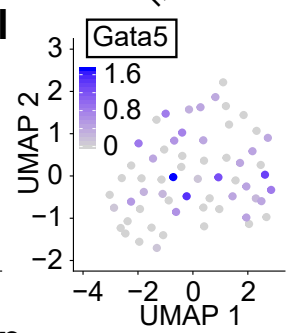
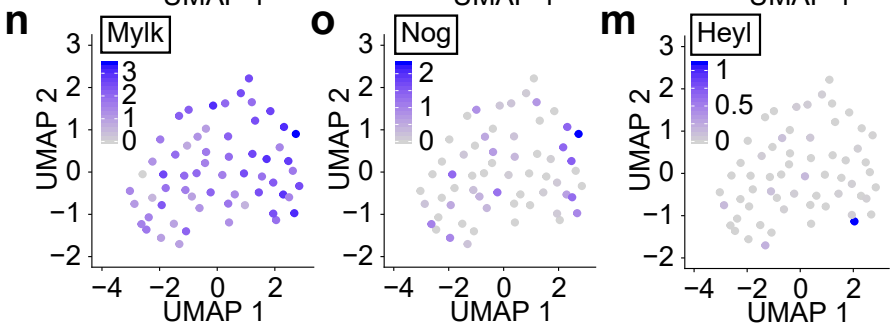

p 3. Speg
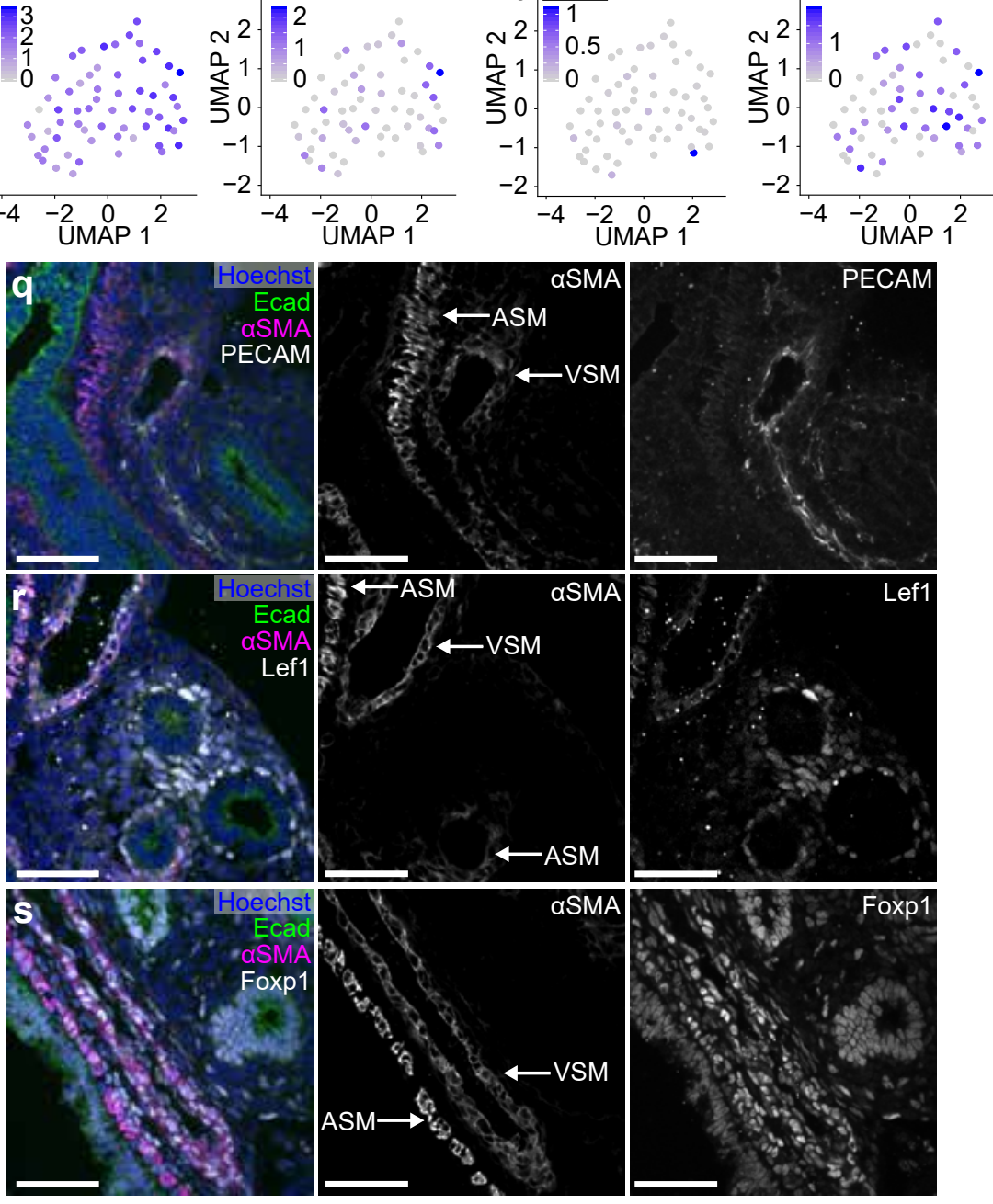


\section{Supplementary Figure 7}
color legend for violin plots
- 0 (sub-epithelial mesenchyme) $\quad 3$ (endothelium)
- 1 (sub-mesothelial mesenchyme) 4 (epithelium)
- 2 (mesothelium)
- 5 (smooth muscle)

CArG(MADS)/PUER-Srf-ChIP-Seq(Sullivan_et_al.)

CCATATIATSGGA

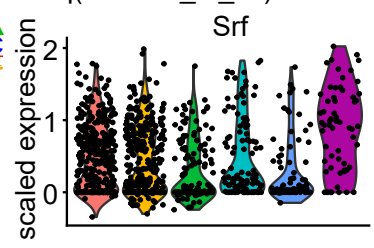

Foxf1(Forkhead)/Lung-Foxf1-ChIP-Seq(GSE77951)

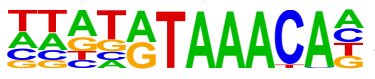

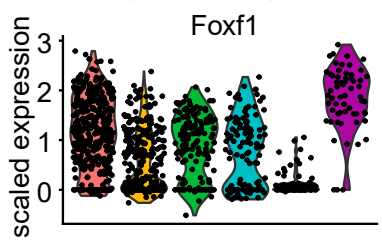

Foxp1(Forkhead)/H9-FOXP1-ChIP-Seq(GSE31006)

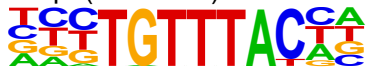

Lef1(HMG)/H1-LEF1-ChIP-Seq(GSE64758)

CCTITGATCT

Tcf7(HMG)/GM12878-TCF7-ChIP-Seq(Encode)

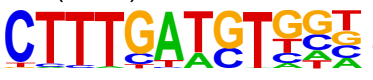

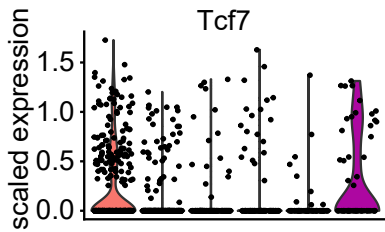

Tcf4(bHLH)/SHSY5Y-TCF4-ChIP-Seq(GSE96915)

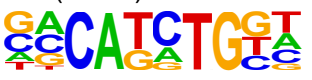

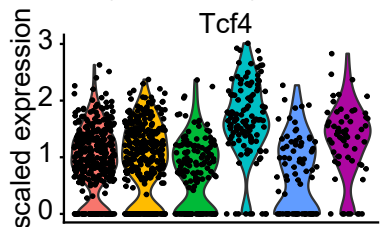

Tcf21(bHLH)/ArterySmoothMuscle-Tcf21-ChIP-Seq(GSE61369)

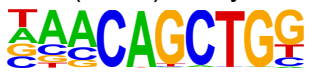

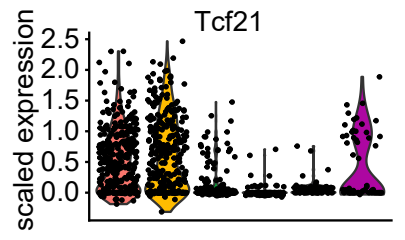

Foxk1(Forkhead)/HEK293-FOXK1-ChIP-Seq(GSE51673)

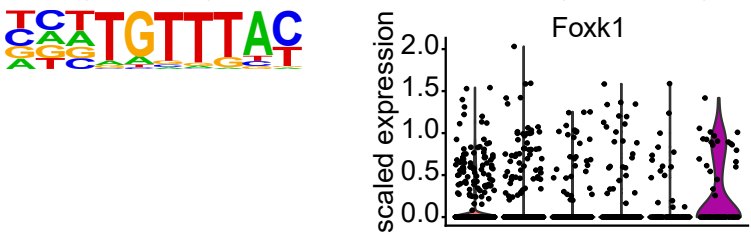

Foxk2(Forkhead)/U2OS-FOXK2-ChIP-Seq(E-MTAB-2204) E्रCATTTTTACAT

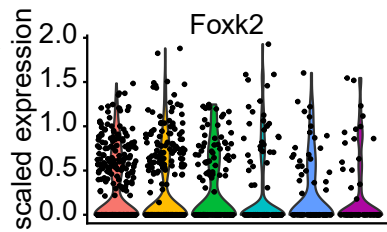

Tbox:Smad(T-box,MAD)/ESCd5-Smad2_3-ChIP-Seq(GSE29422)

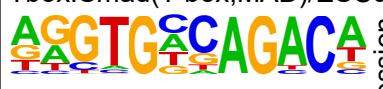
Smad3

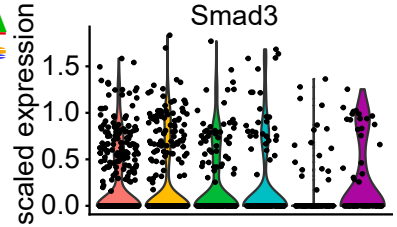

Smad2(MAD)/ES-SMAD2-ChIP-Seq(GSE29422) CTETCTCT TG

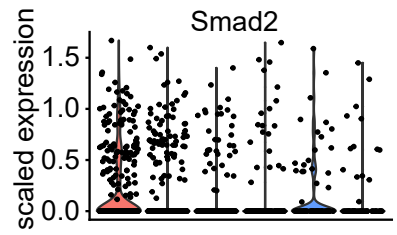

Smad4(MAD)/ESC-SMAD4-ChIP-Seq(GSE29422)

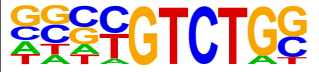

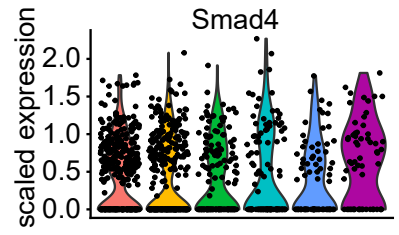

Tead(TEA)/Fibroblast-PU.1-ChIP-Seq(Unpublished)/Homer

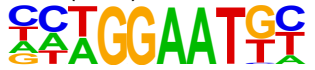

Tead1(TEAD)/HepG2-TEAD1-ChIP-Seq(Encode) СC수을TCCA

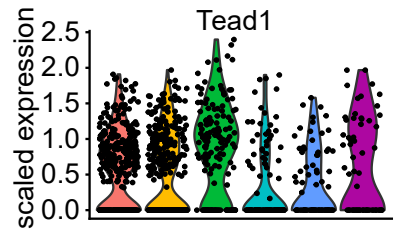

Tead3(TEA)/HepG2-TEAD3-ChIP-Seq(Encode) T्रू:ACATTCCA

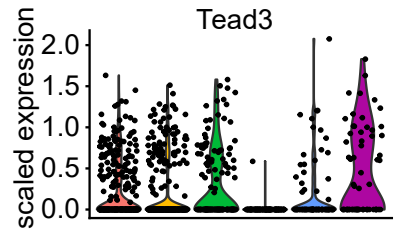

Tead4(TEA)/Tropoblast-Tead4-ChIP-Seq(GSE37350)

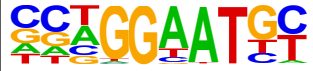

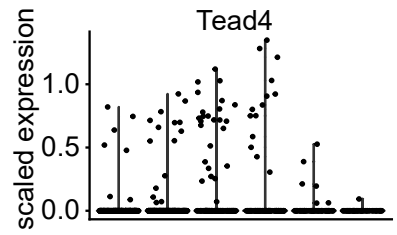

c-Myc(bHLH)/mES-cMyc-ChIP-Seq(GSE11431)

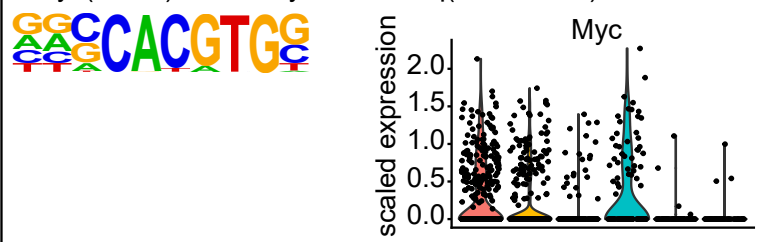

n-Myc(bHLH)/mES-nMyc-ChIP-Seq(GSE11431)

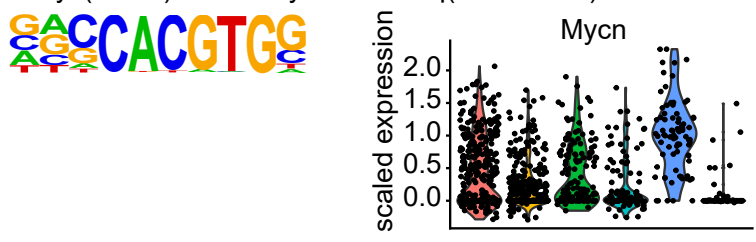


Supplementary Figure 8

a
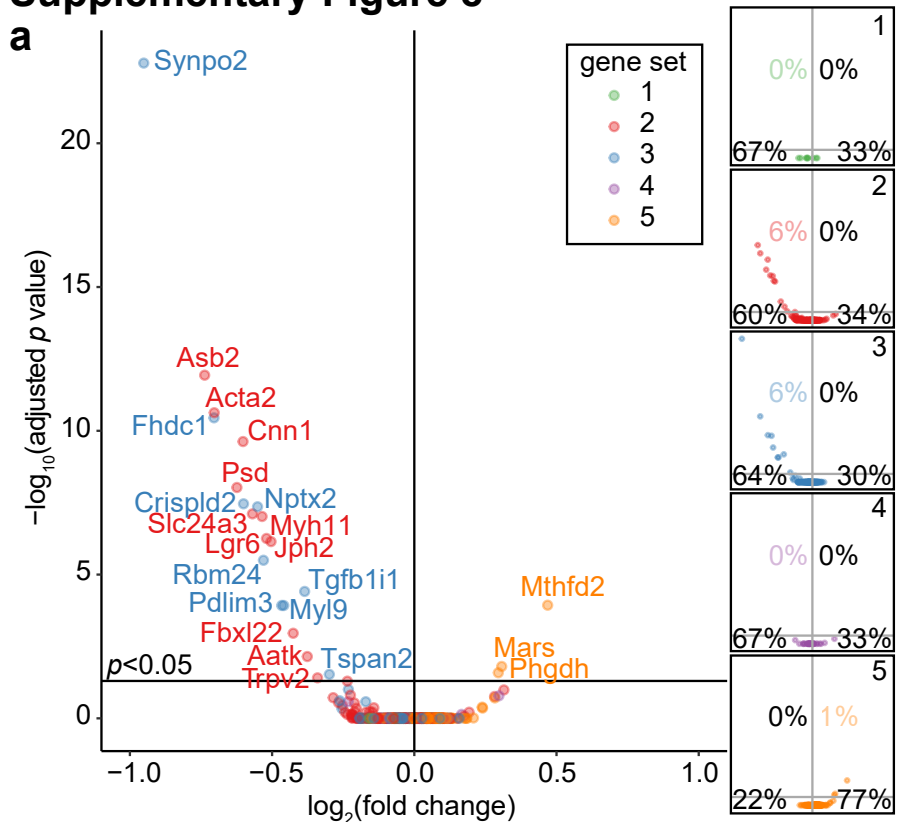

c

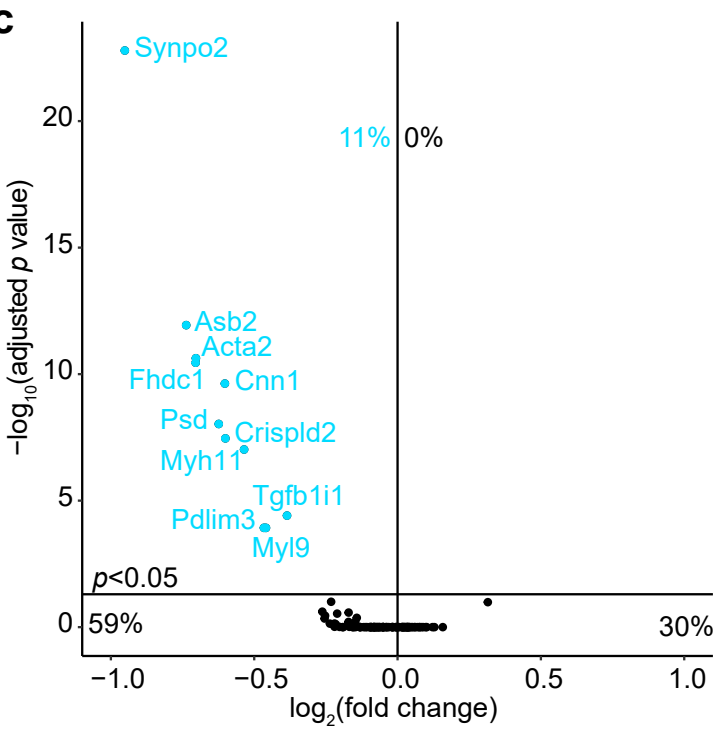

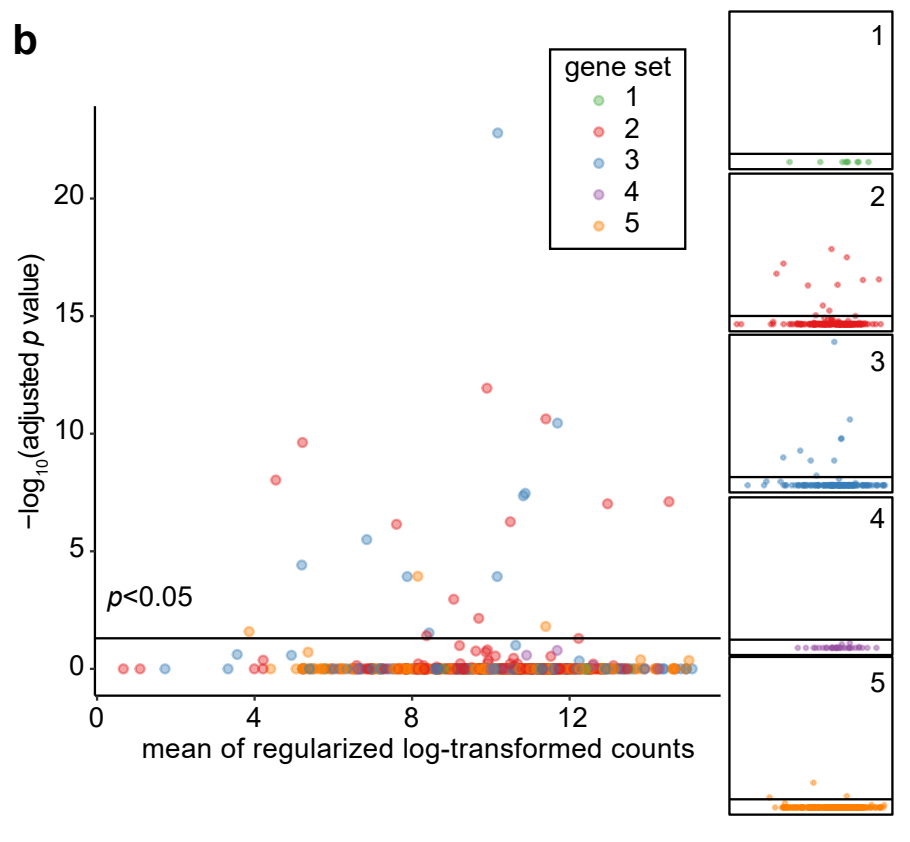

d

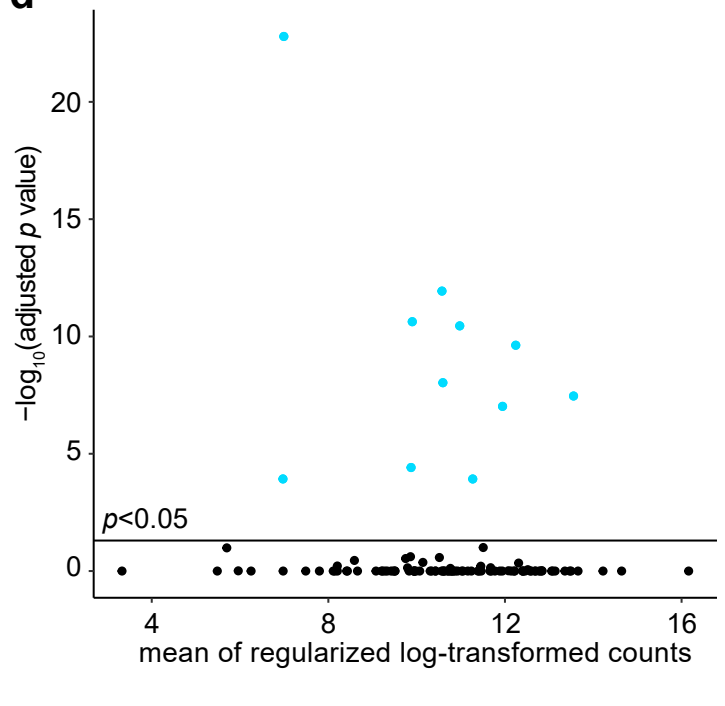

